\title{
Development of an Efficient Synthetic Process for Broflanilide
}

Chunyan Luo, ${ }^{\dagger, \ddagger}$ Qiong Xu, ${ }^{* \dagger}$ Chaoqun Huang, ${ }^{*, \neq}$ Liangming Luo, Jintao Zhu, Rong Zhang, ${ }^{\ddagger}$ Geng Huang, ${ }^{\dagger}$ Dulin Yin ${ }^{*}$,

$\dagger$ National \& Local Joint Engineering Laboratory for New Petro-chemical Materials and Fine Utilization of Resources, College of Chemistry and Chemical Engineering, Hunan Normal University, 36 Lushan Rd., Changsha 410081, Hunan, P. R. China

†ChangSha Jiaqiao Biological Technology Co. Ltd., 186 Guyuan Rd., Changsha 410205, Hunan, P. R. China

\section{Supporting Information}

\section{Table of Contents:}

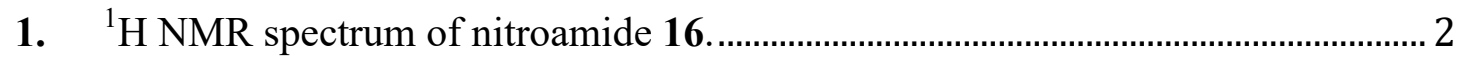

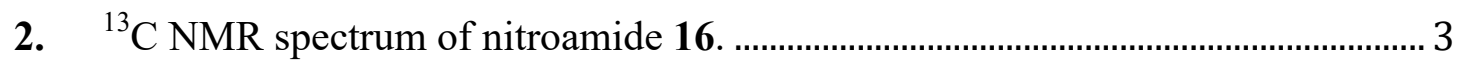

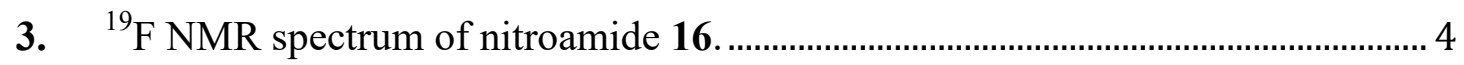

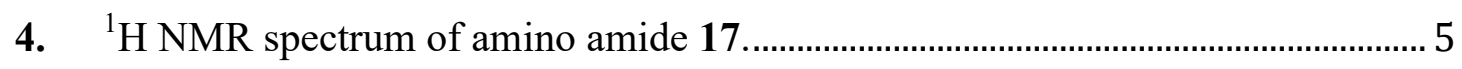

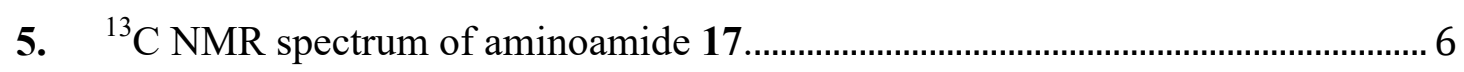

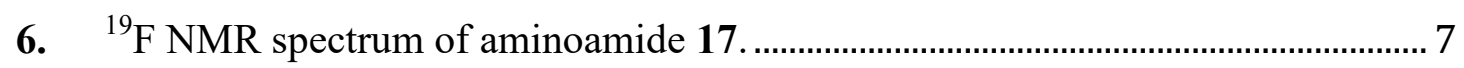

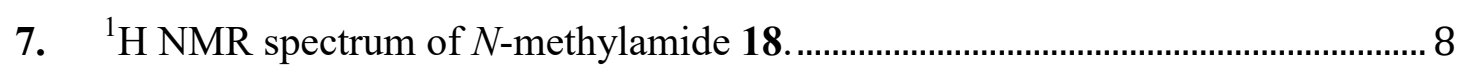

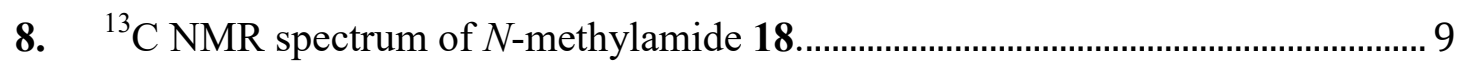

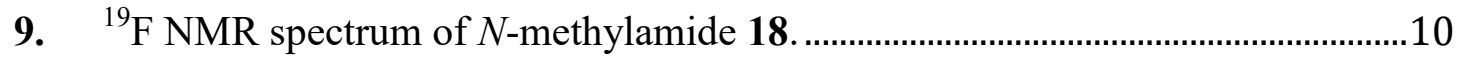

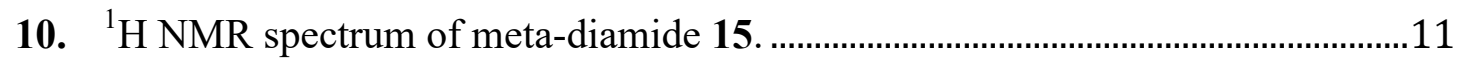

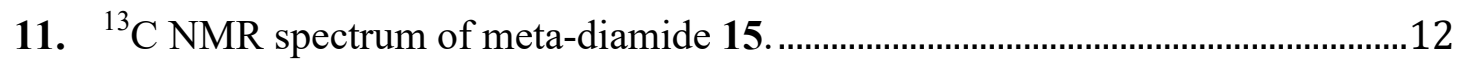


12. ${ }^{19} \mathrm{~F}$ NMR spectrum of meta-diamide $\mathbf{1 5}$.

13. ${ }^{1} \mathrm{H}$ NMR spectrum of broflanilide (1) ……...................................................... 14

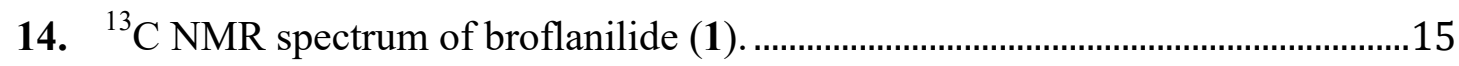

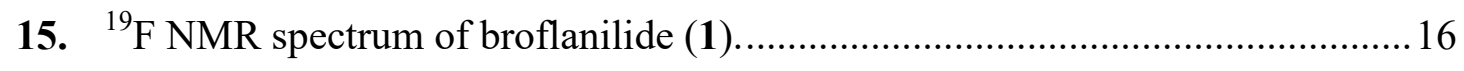

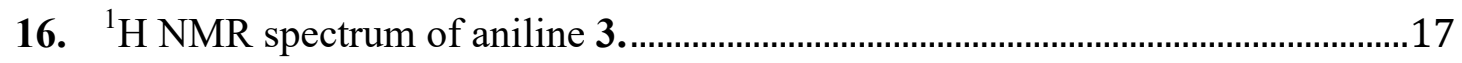

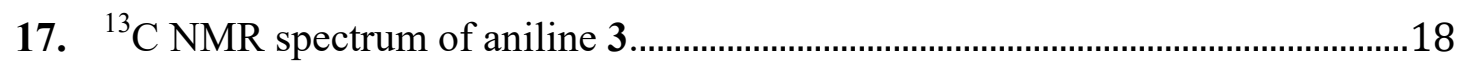

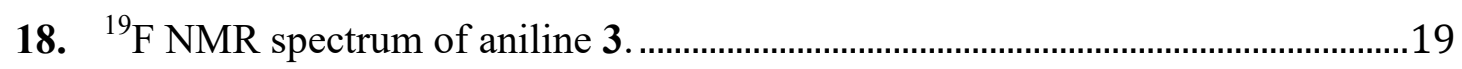

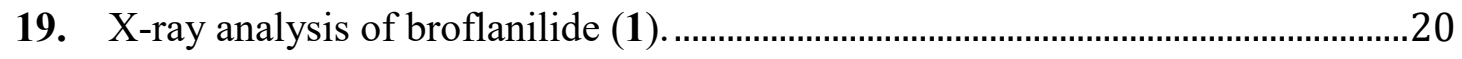

1. ${ }^{1} \mathrm{H}$ NMR spectrum of nitroamide 16.

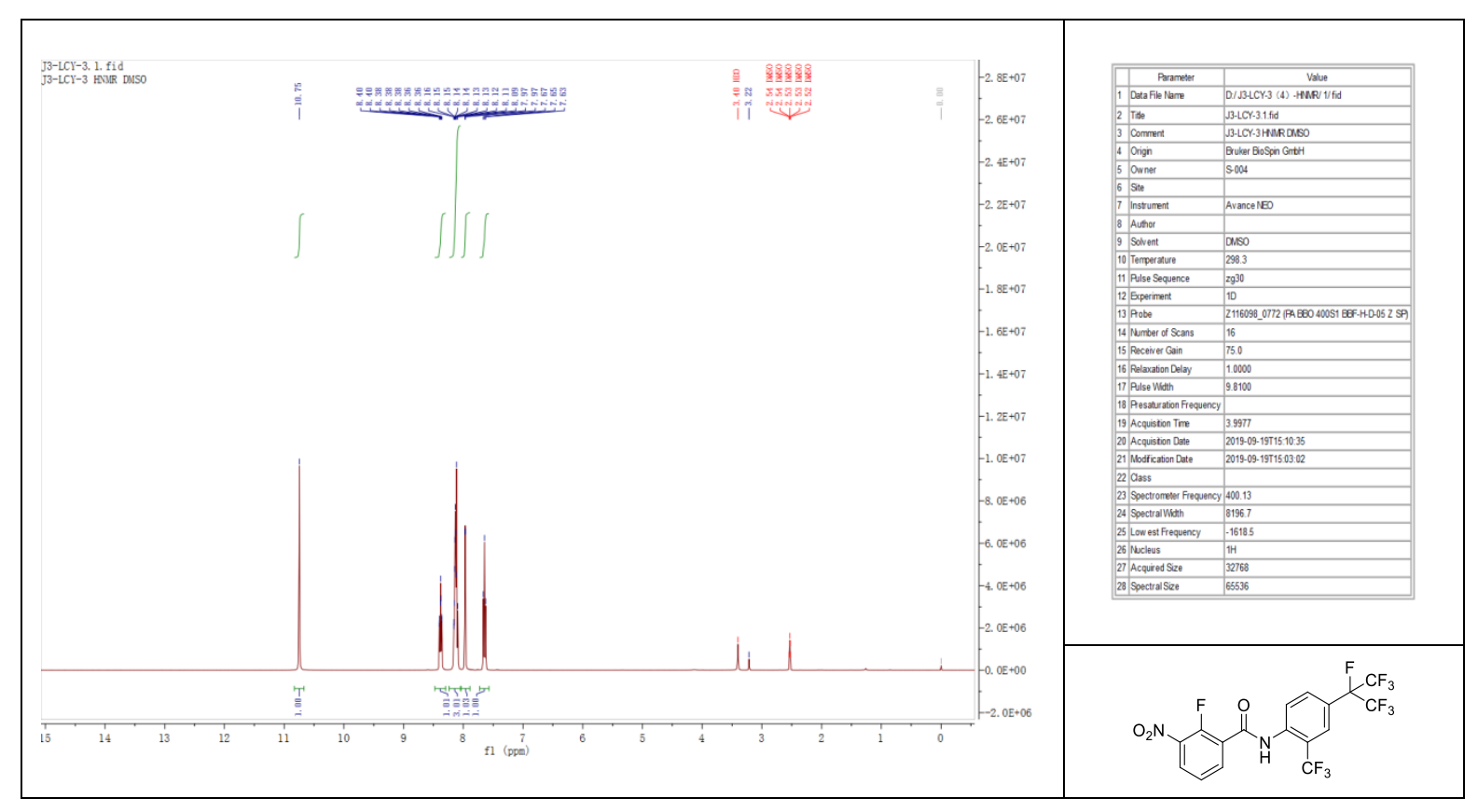


2. ${ }^{13} \mathrm{C}$ NMR spectrum of nitroamide $\mathbf{1 6}$.

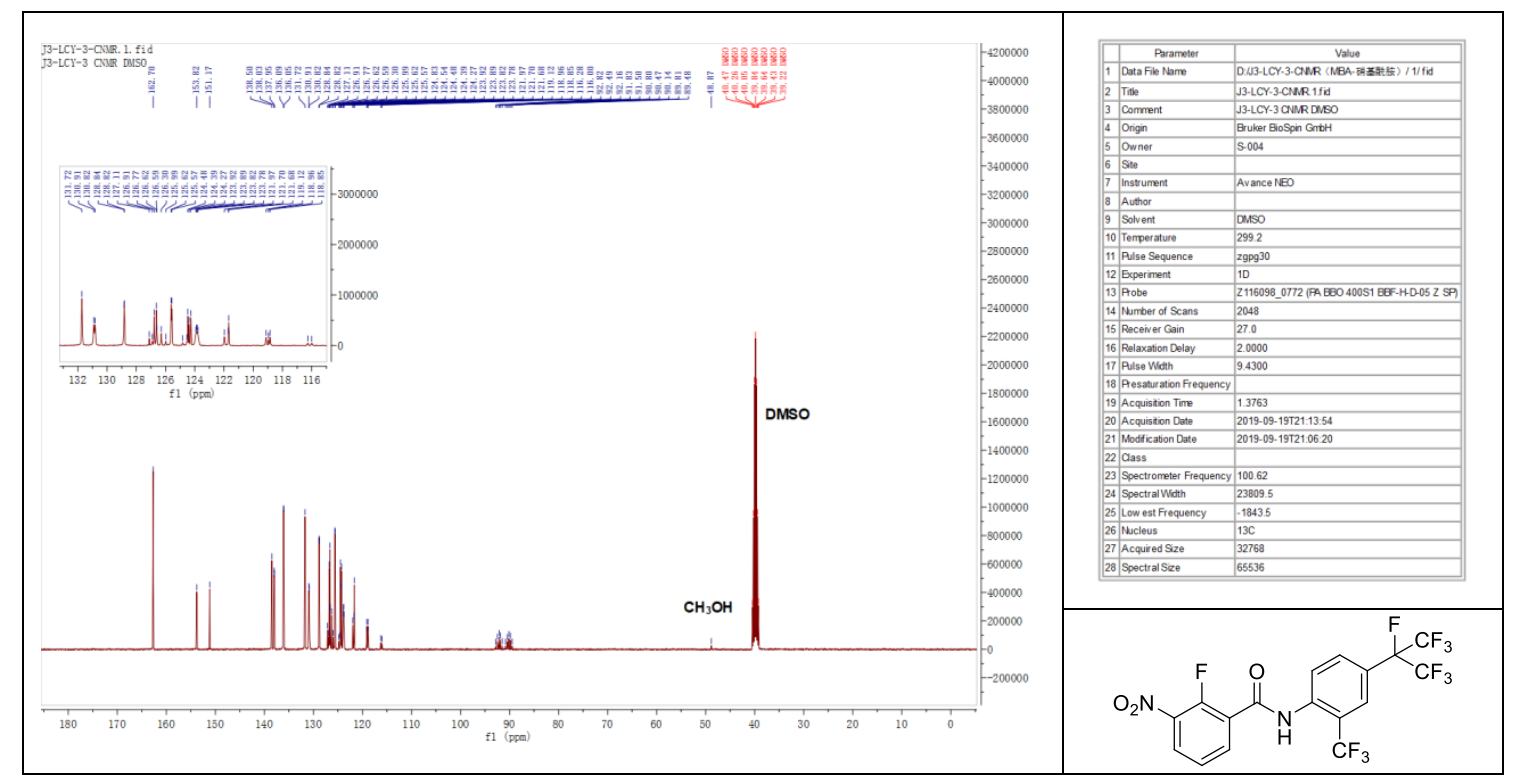


3. ${ }^{19}$ F NMR spectrum of nitroamide $\mathbf{1 6}$.

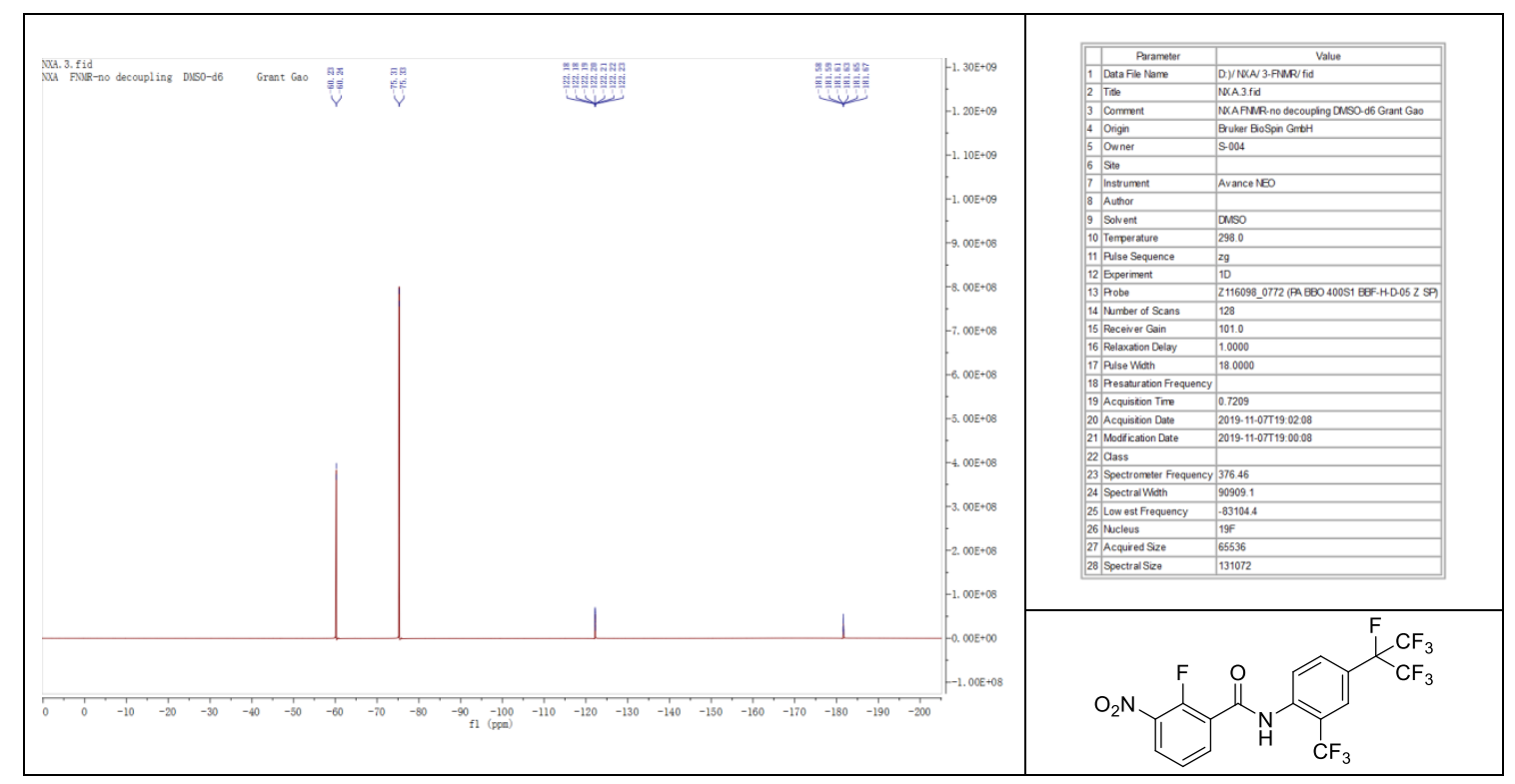


4. ${ }^{1} \mathrm{H}$ NMR spectrum of amino amide $\mathbf{1 7}$.

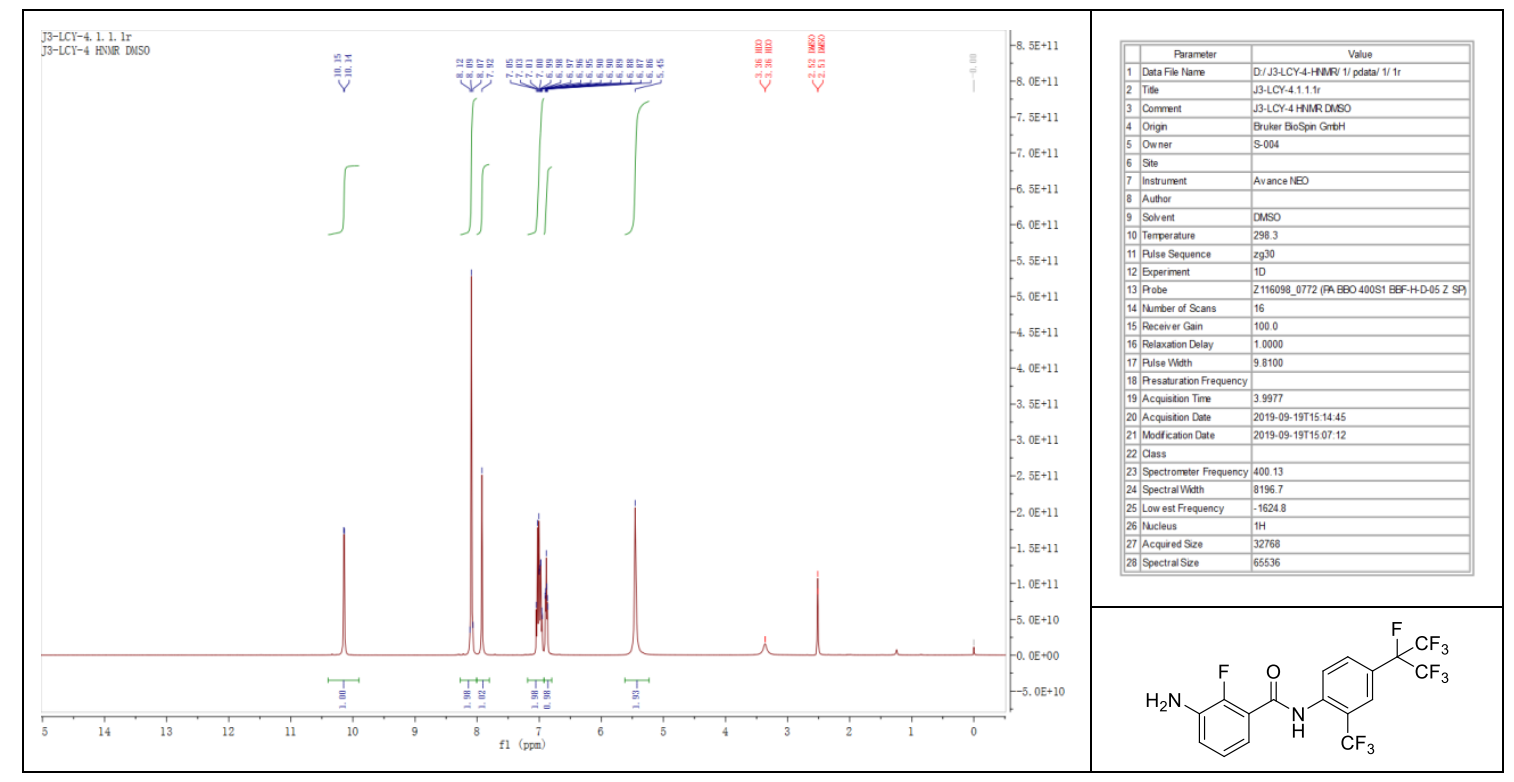


5. ${ }^{13} \mathrm{C}$ NMR spectrum of aminoamide $\mathbf{1 7}$.

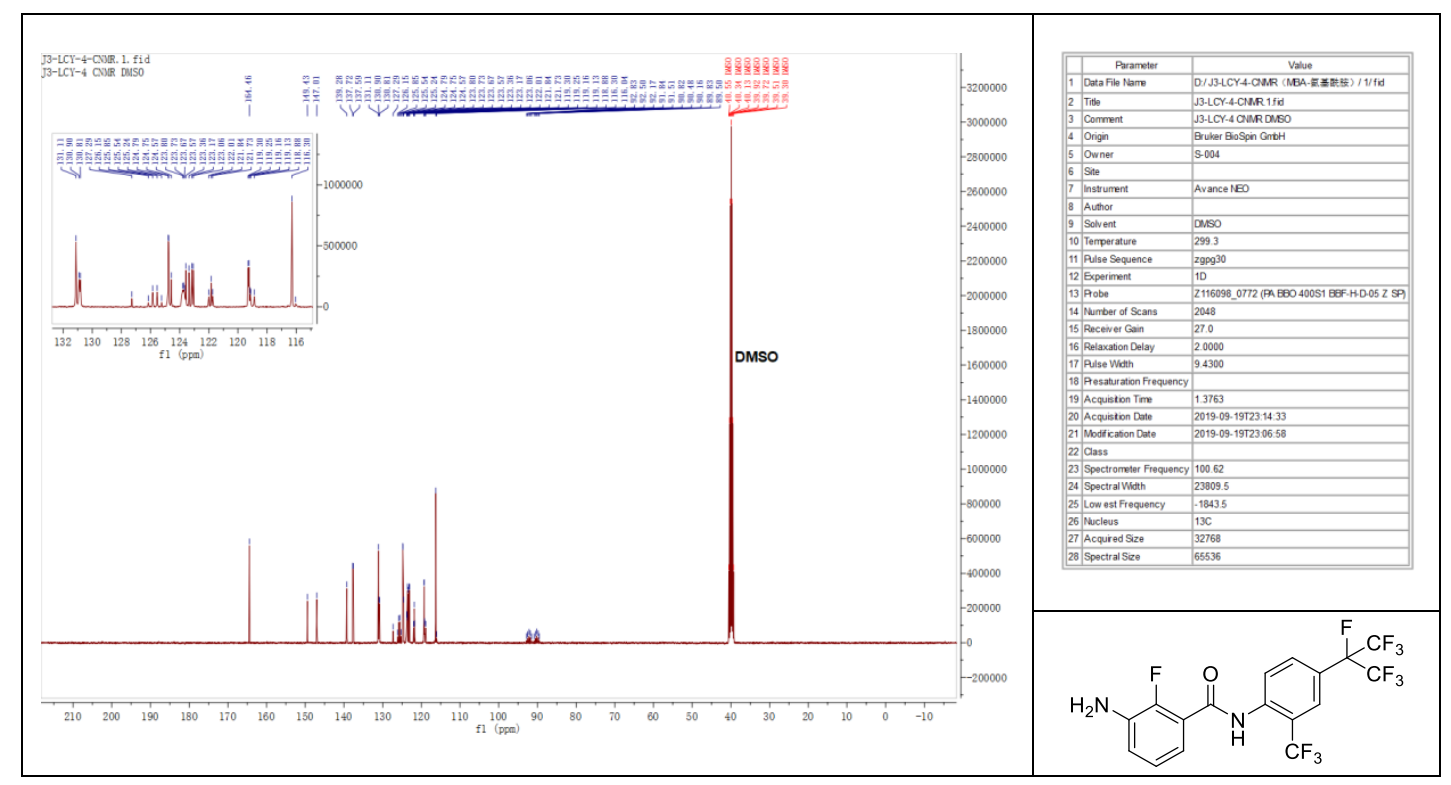


6. ${ }^{19}$ F NMR spectrum of aminoamide $\mathbf{1 7}$.

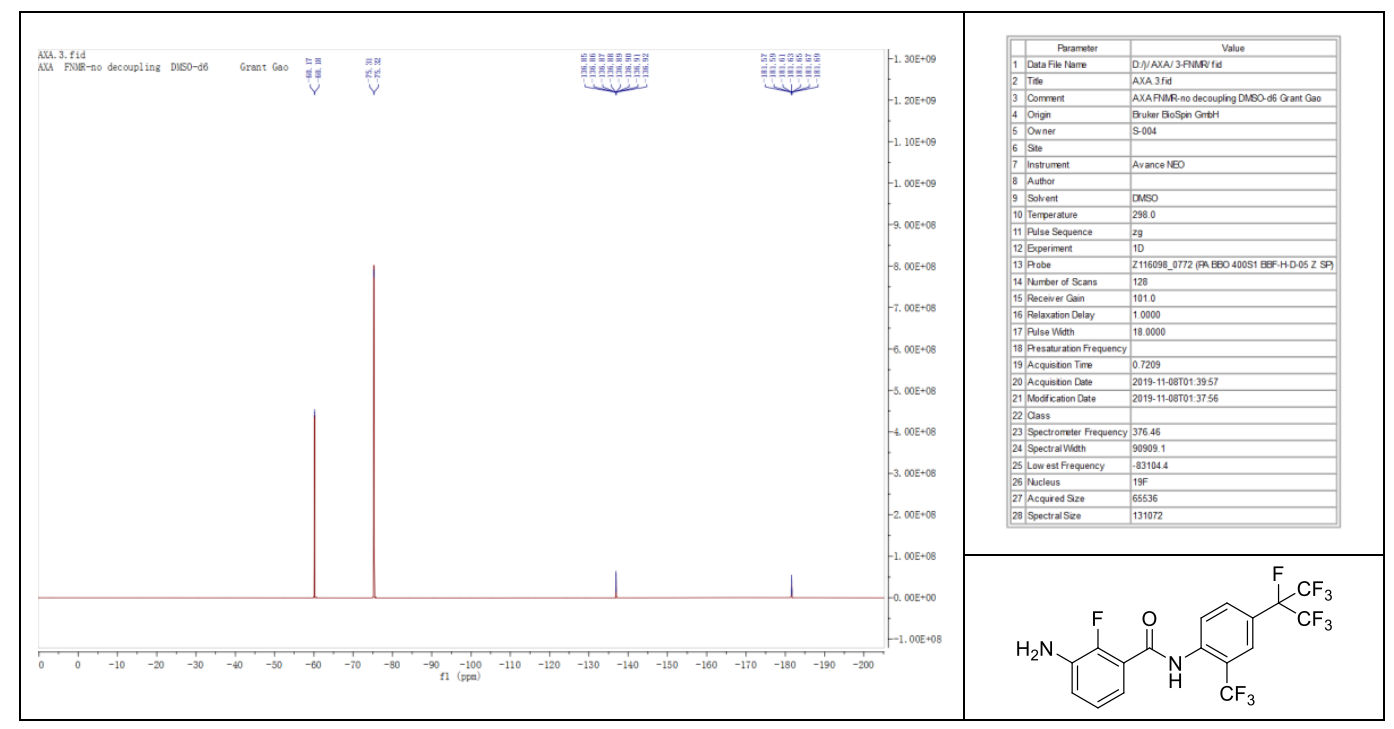


7. ${ }^{1} \mathrm{H}$ NMR spectrum of $N$-methylamide $\mathbf{1 8}$.

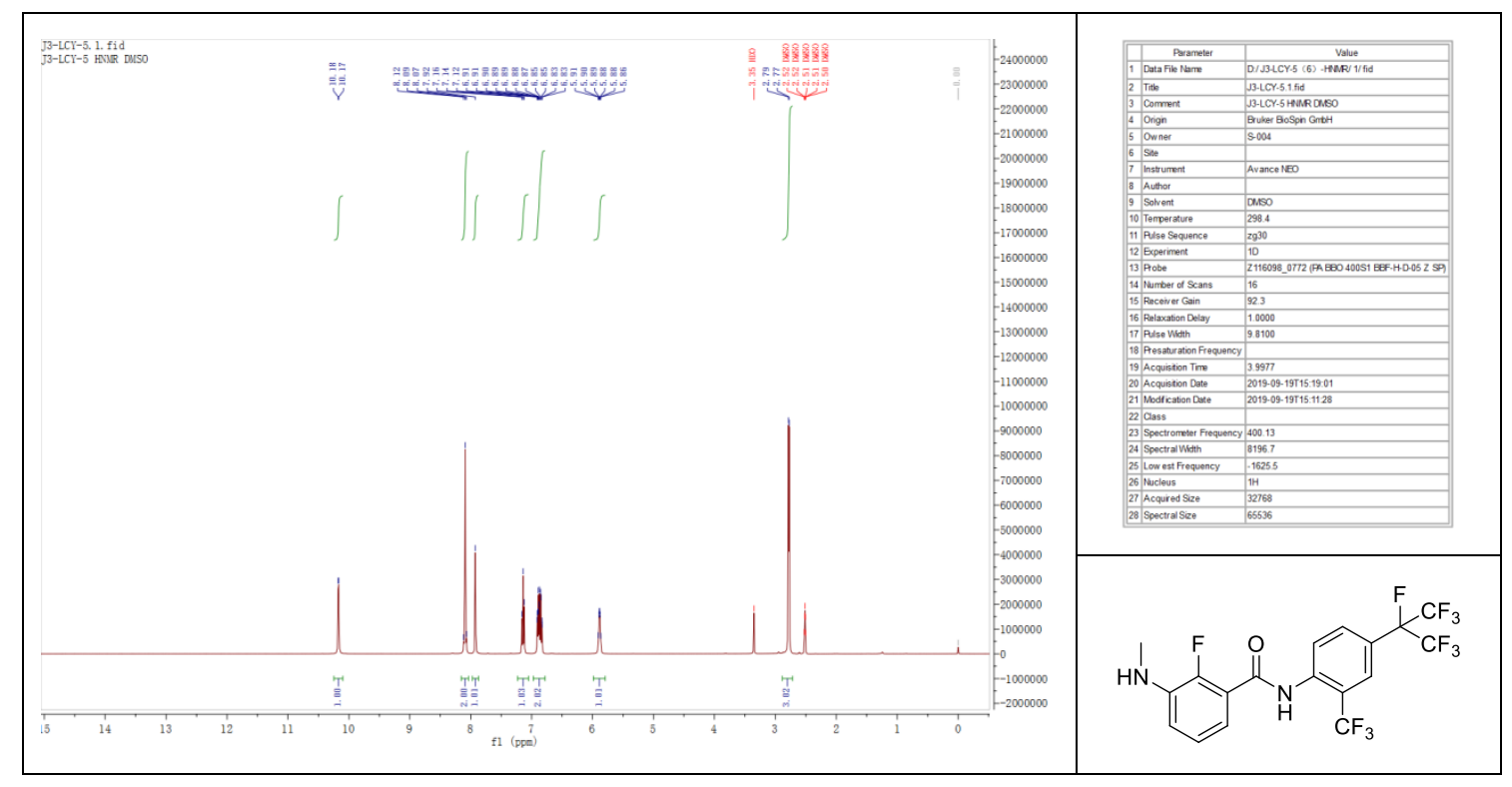


8. ${ }^{13} \mathrm{C}$ NMR spectrum of $N$-methylamide 18 .

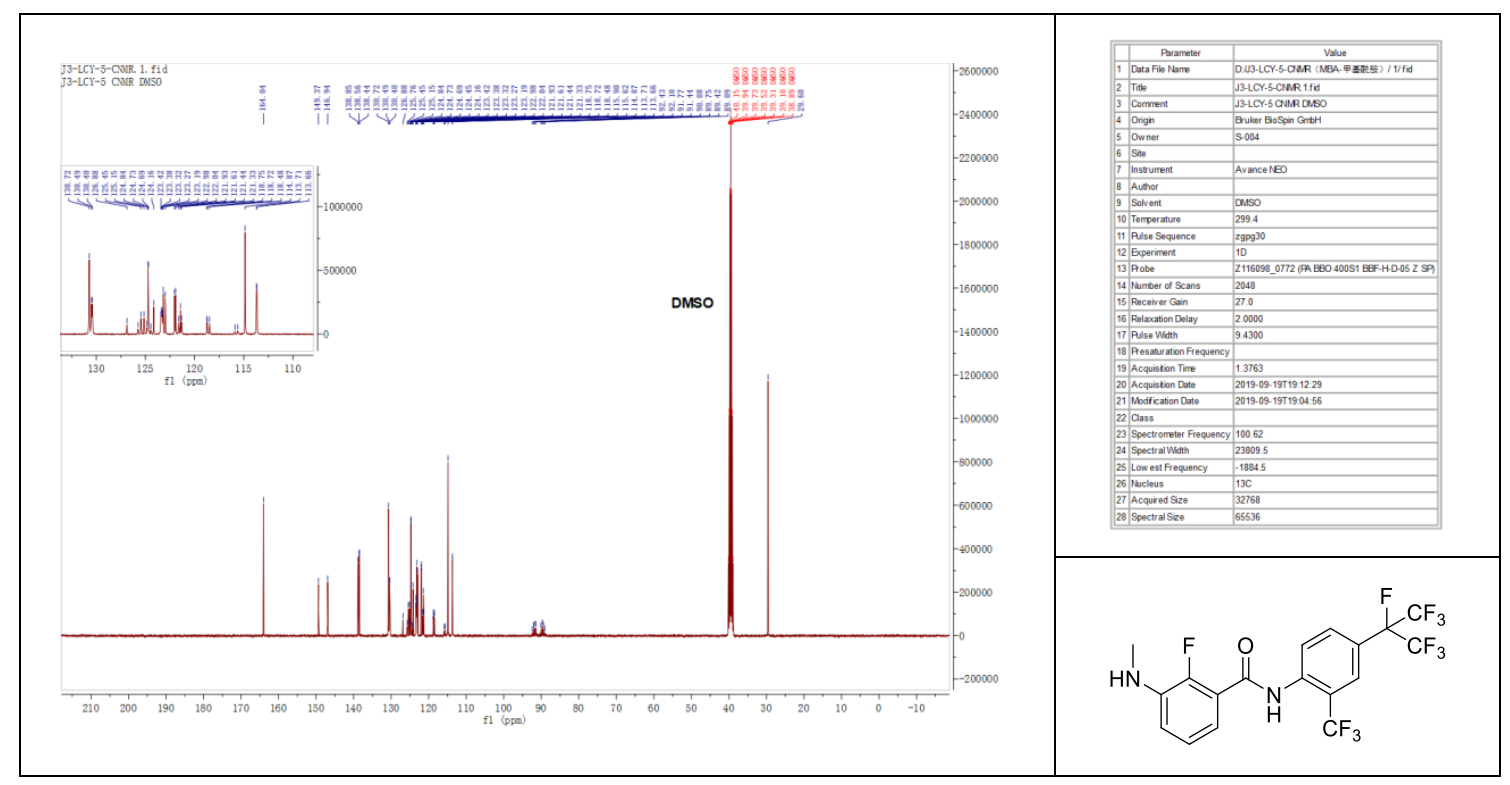


9. ${ }^{19}$ F NMR spectrum of $N$-methylamide $\mathbf{1 8}$.

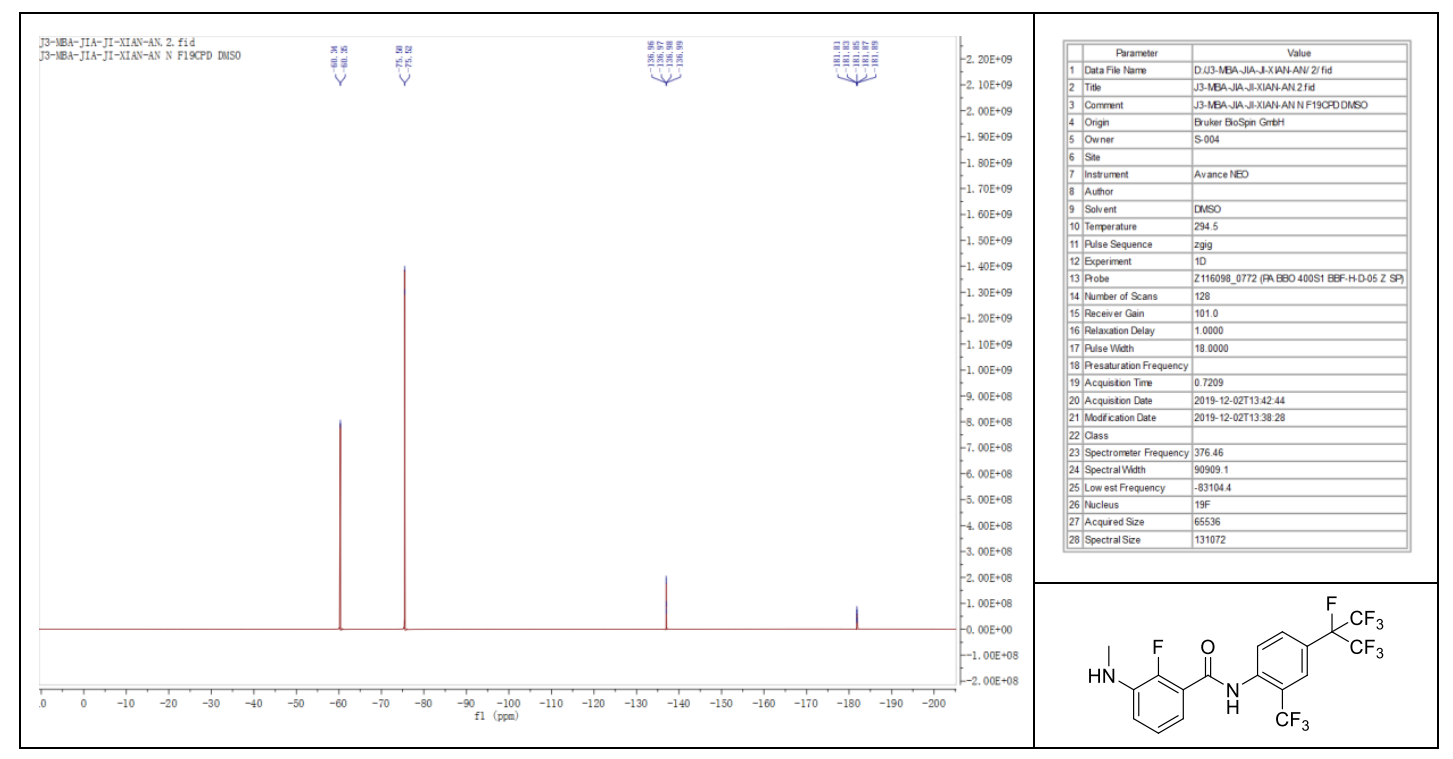


10. ${ }^{1} \mathrm{H}$ NMR spectrum of meta-diamide $\mathbf{1 5}$.

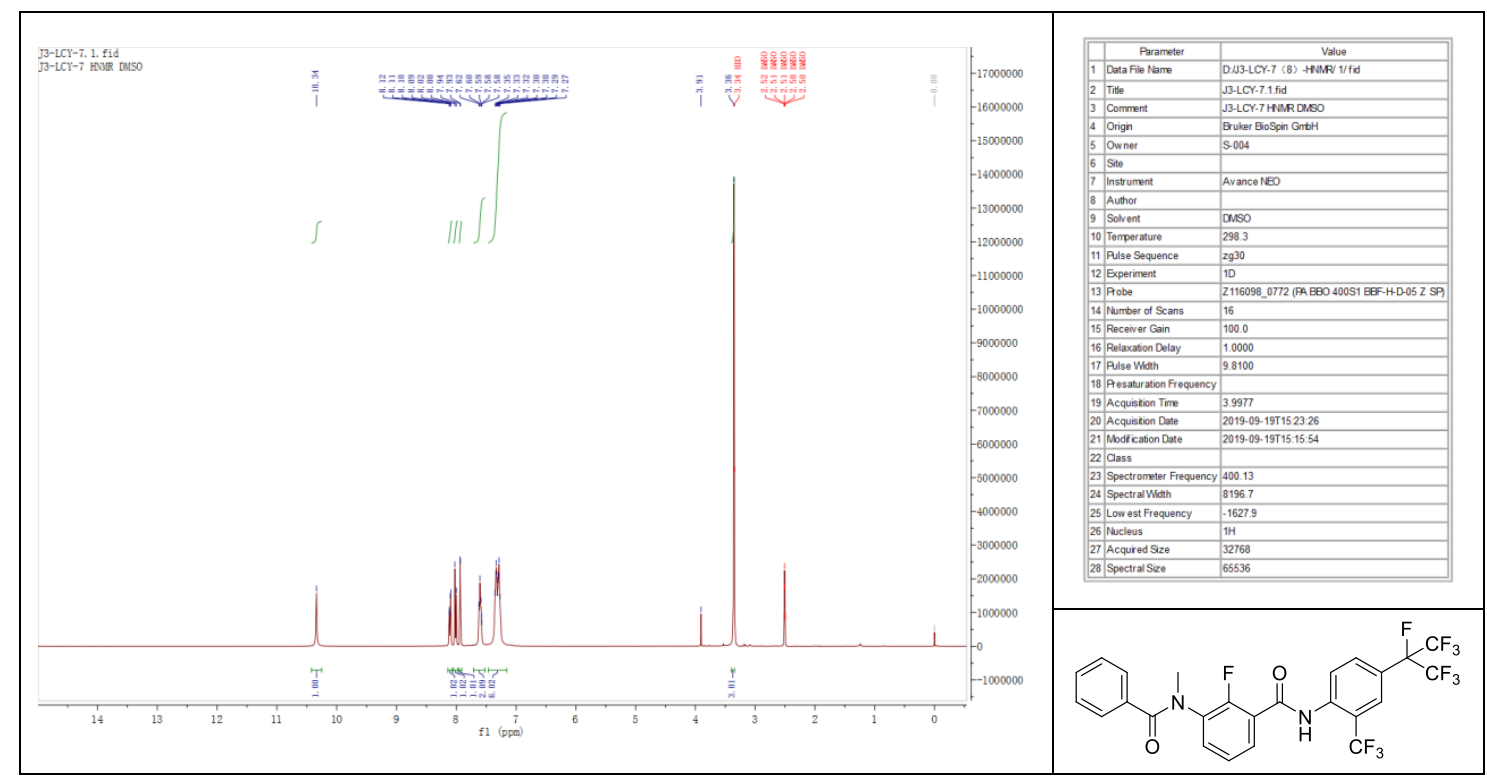


11. ${ }^{13} \mathrm{C}$ NMR spectrum of meta-diamide $\mathbf{1 5}$.

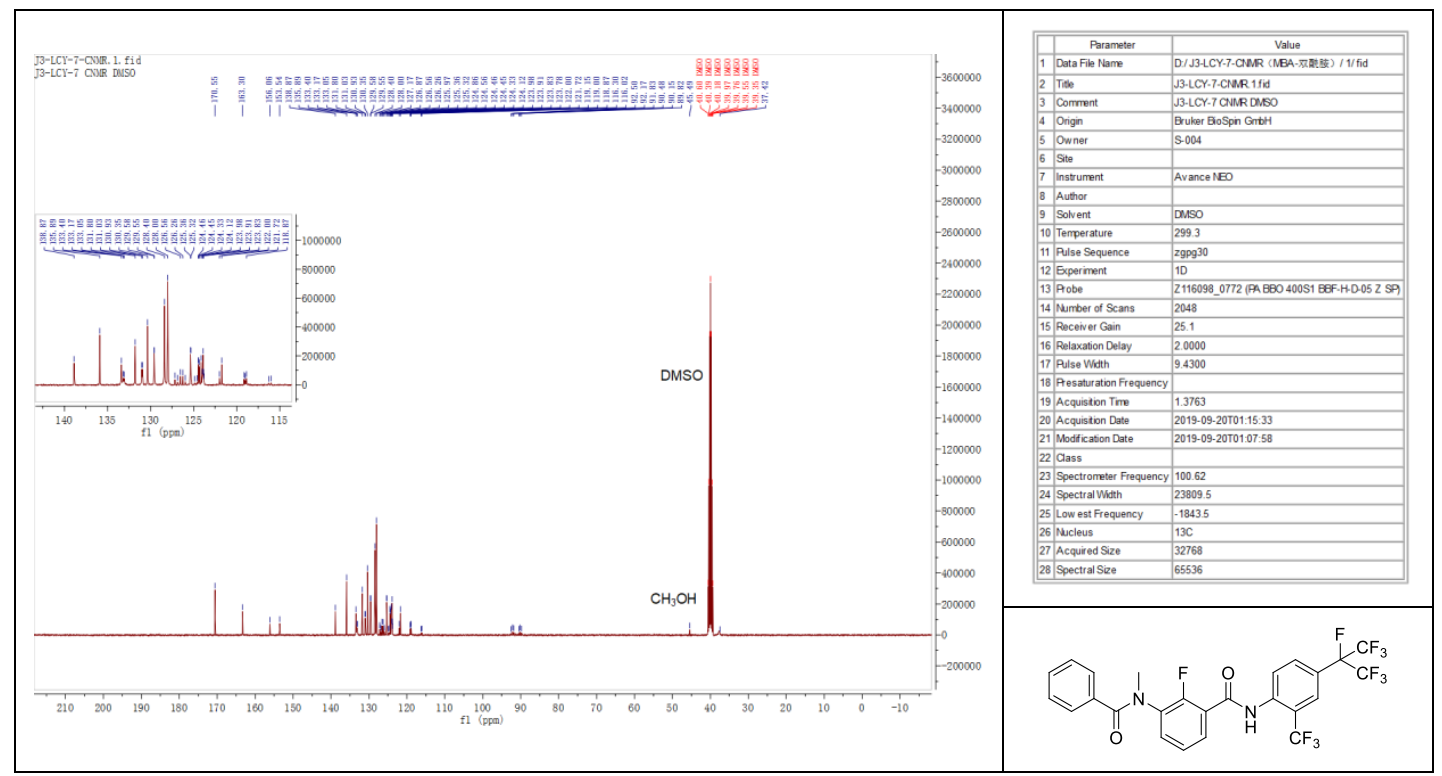


12. ${ }^{19}$ F NMR spectrum of meta-diamide $\mathbf{1 5}$.

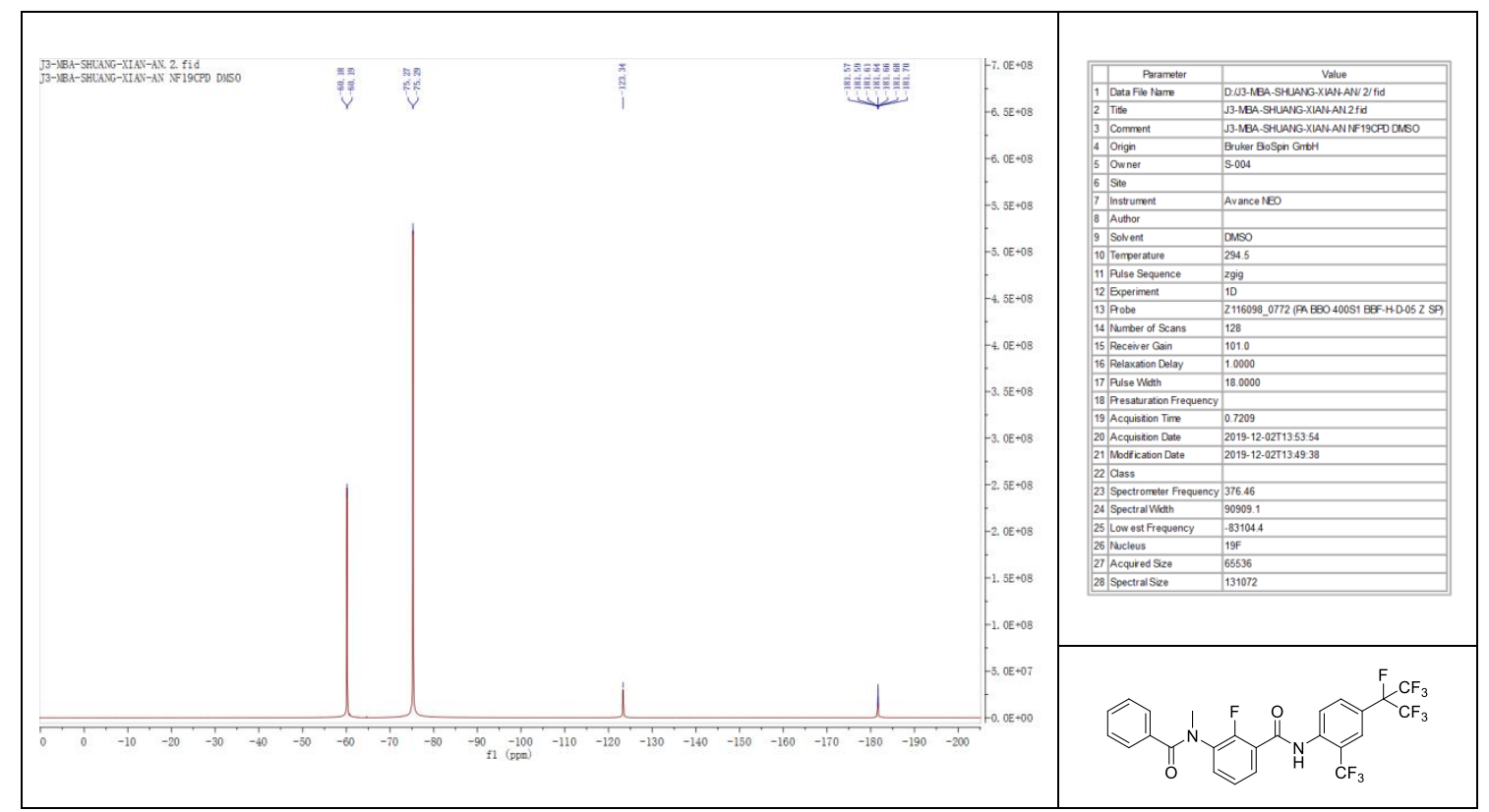


13. ${ }^{1} \mathrm{H}$ NMR spectrum of broflanilide (1).

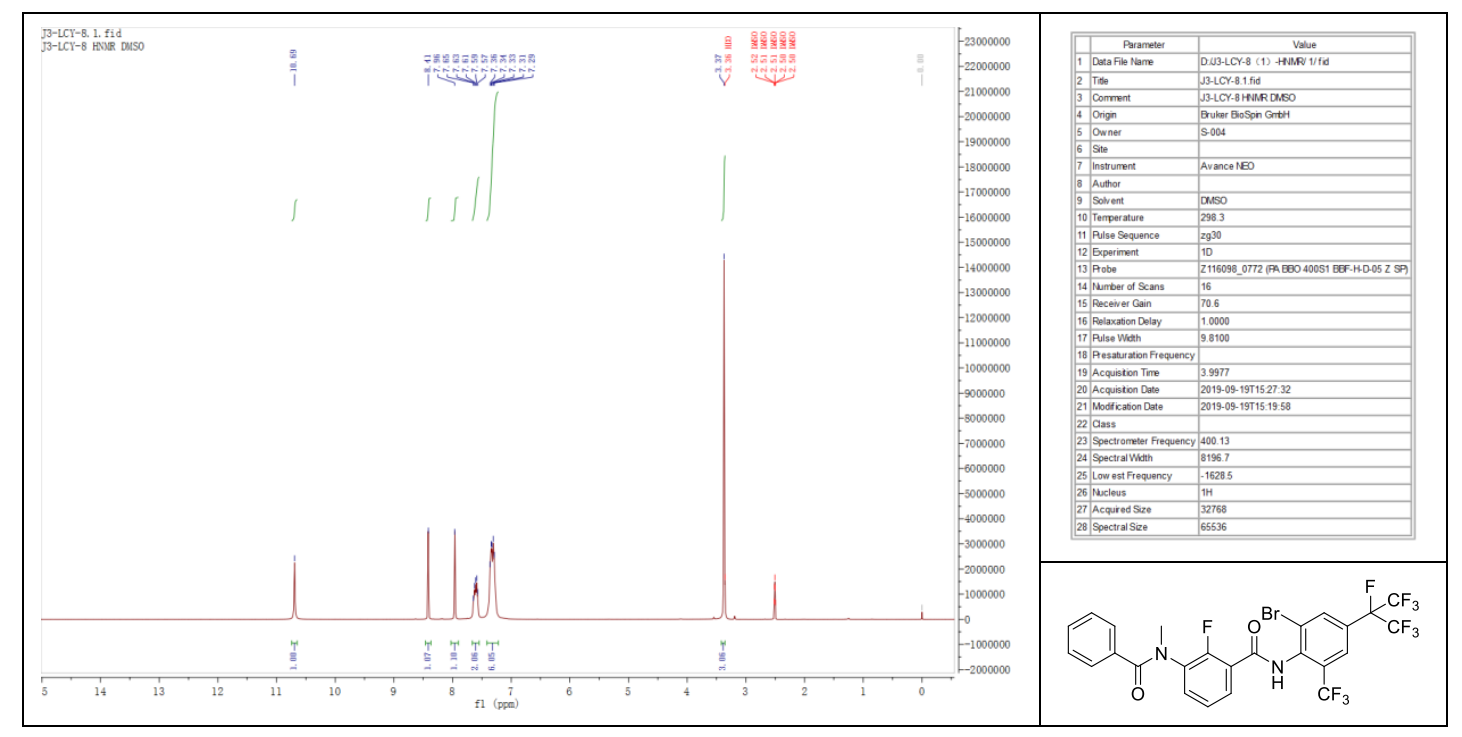


14. ${ }^{13} \mathrm{C}$ NMR spectrum of broflanilide (1).

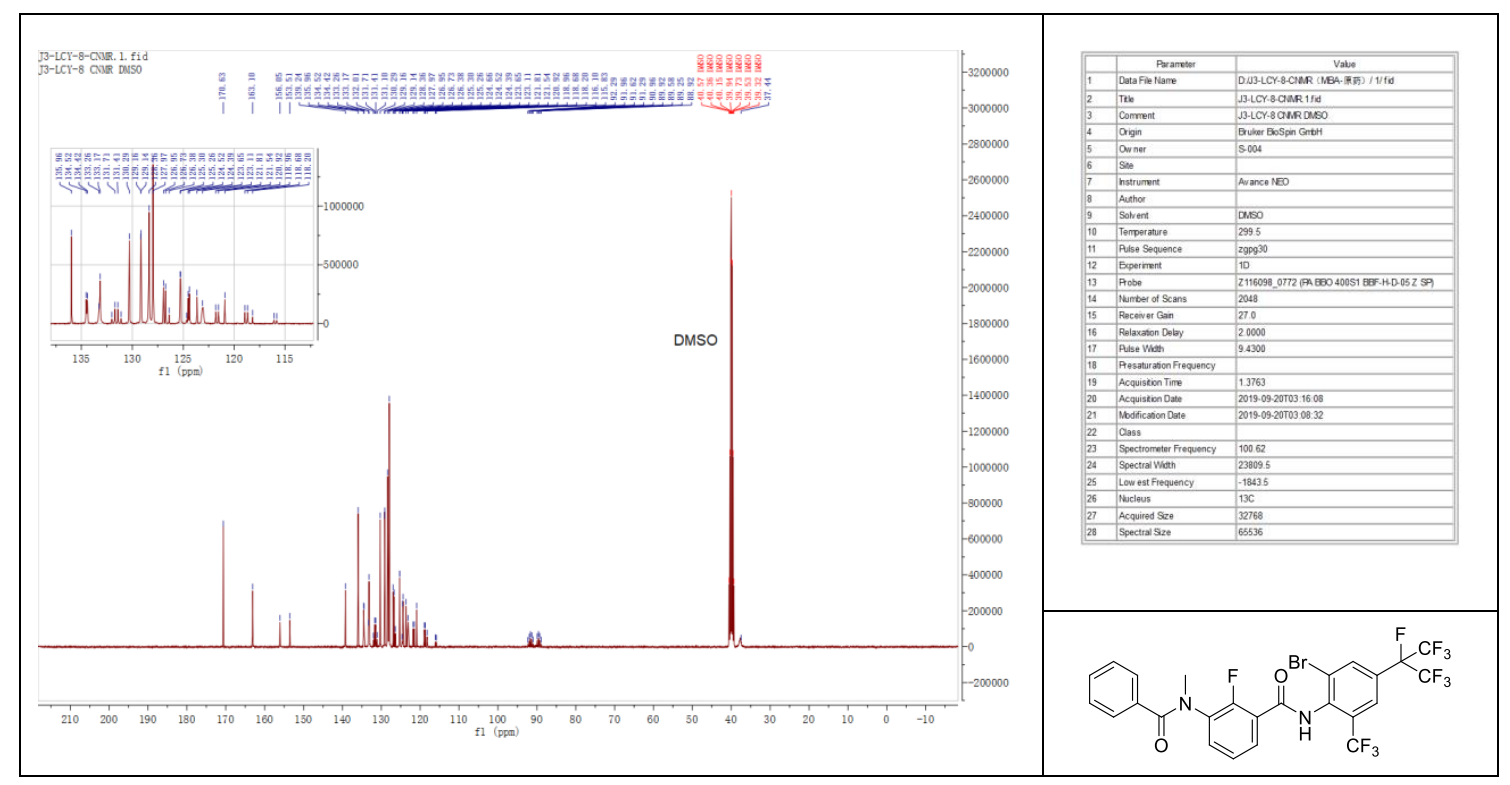


15. ${ }^{19} \mathrm{~F}$ NMR spectrum of broflanilide (1).

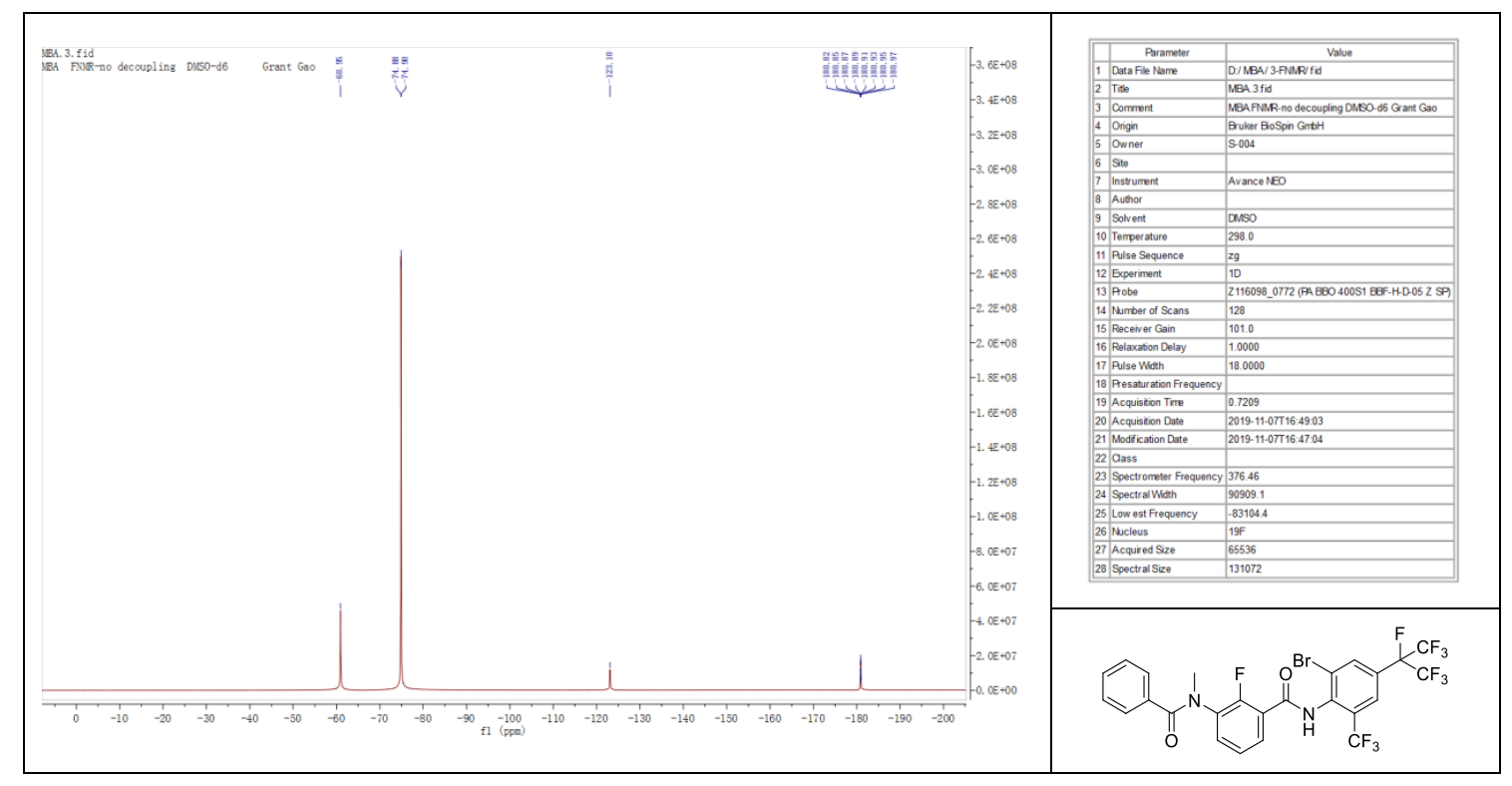


16. ${ }^{1} \mathrm{H}$ NMR spectrum of aniline 3 .

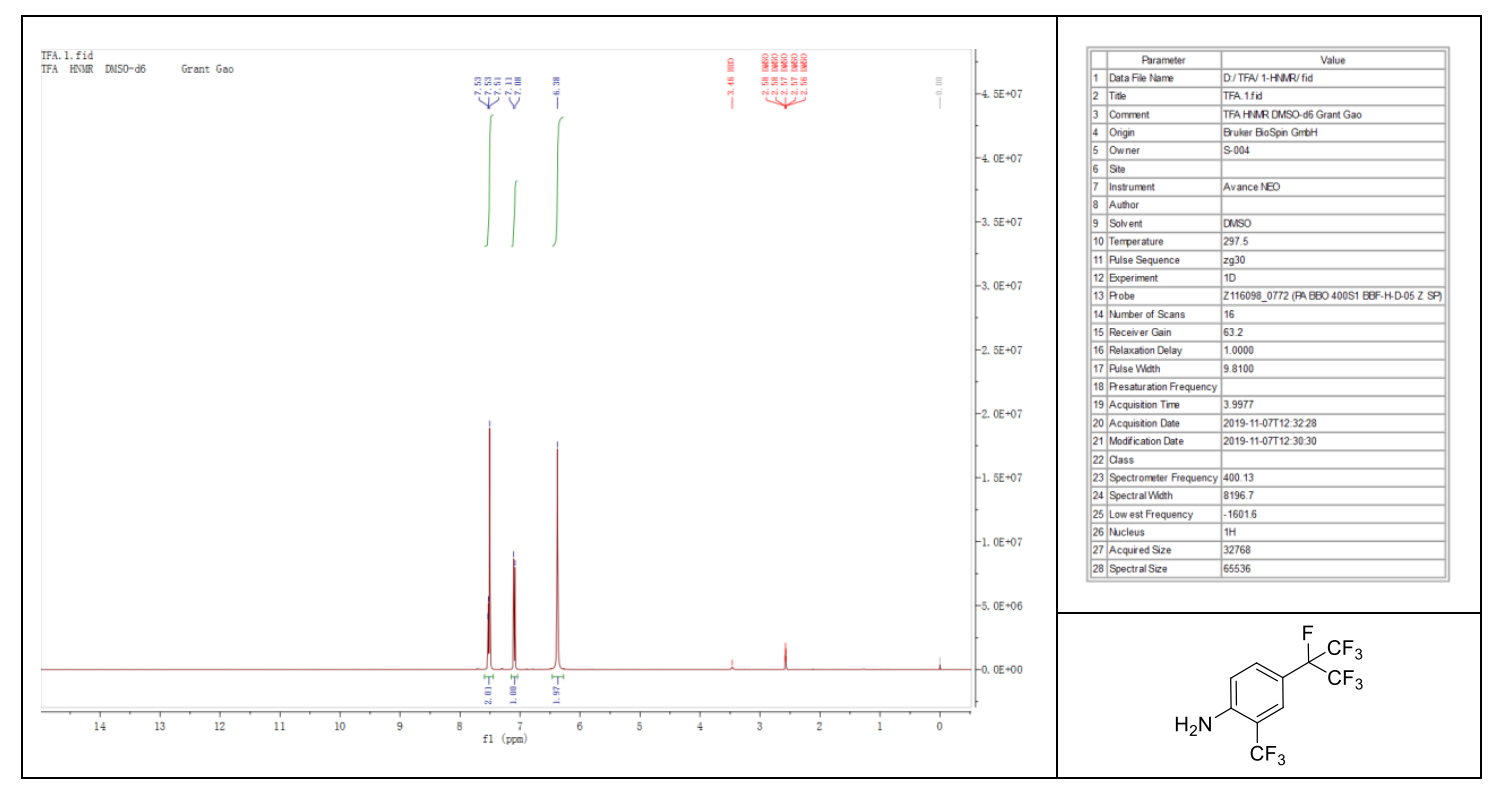


17. ${ }^{13} \mathrm{C}$ NMR spectrum of aniline 3 .

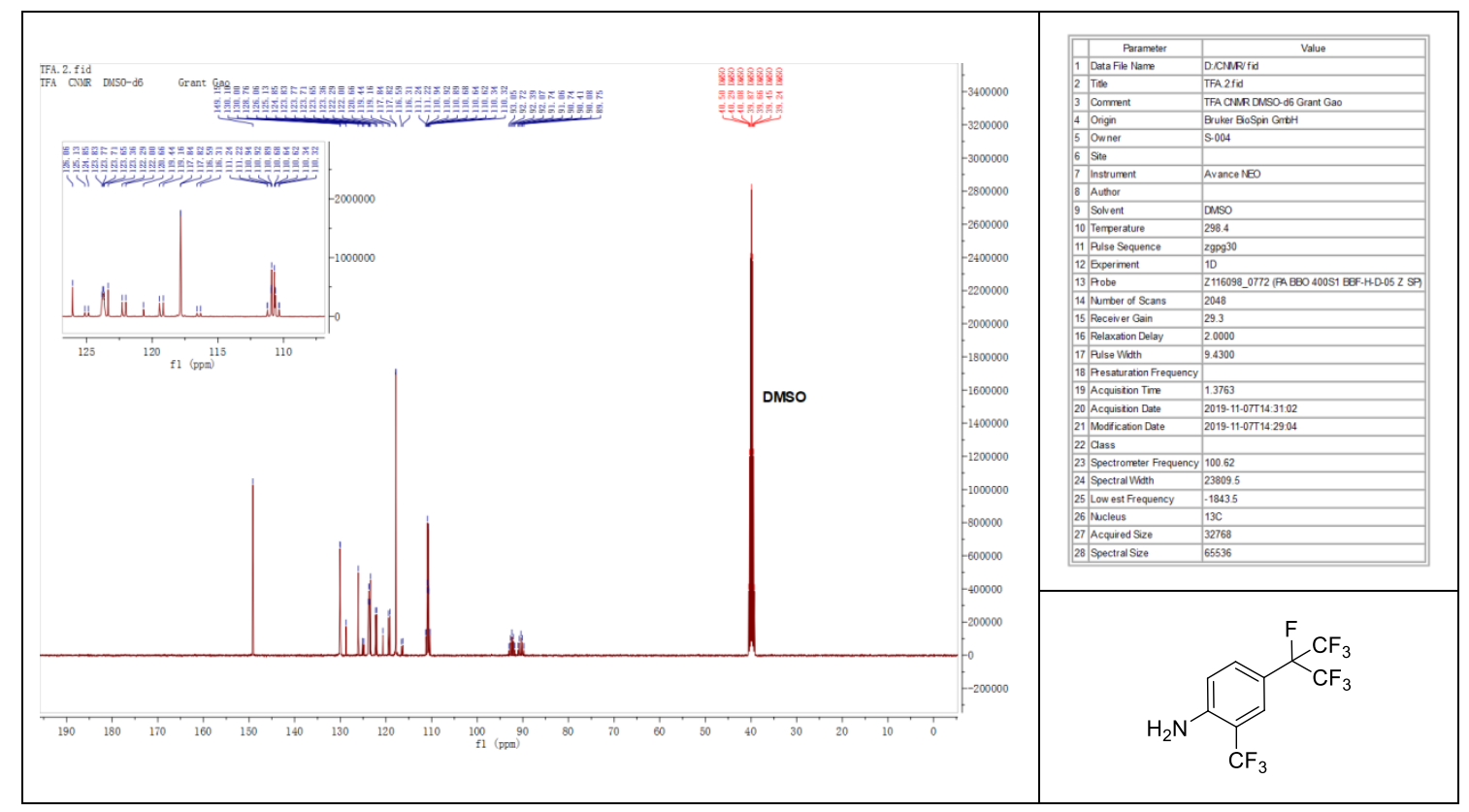


18. ${ }^{19} \mathrm{~F}$ NMR spectrum of aniline 3 .

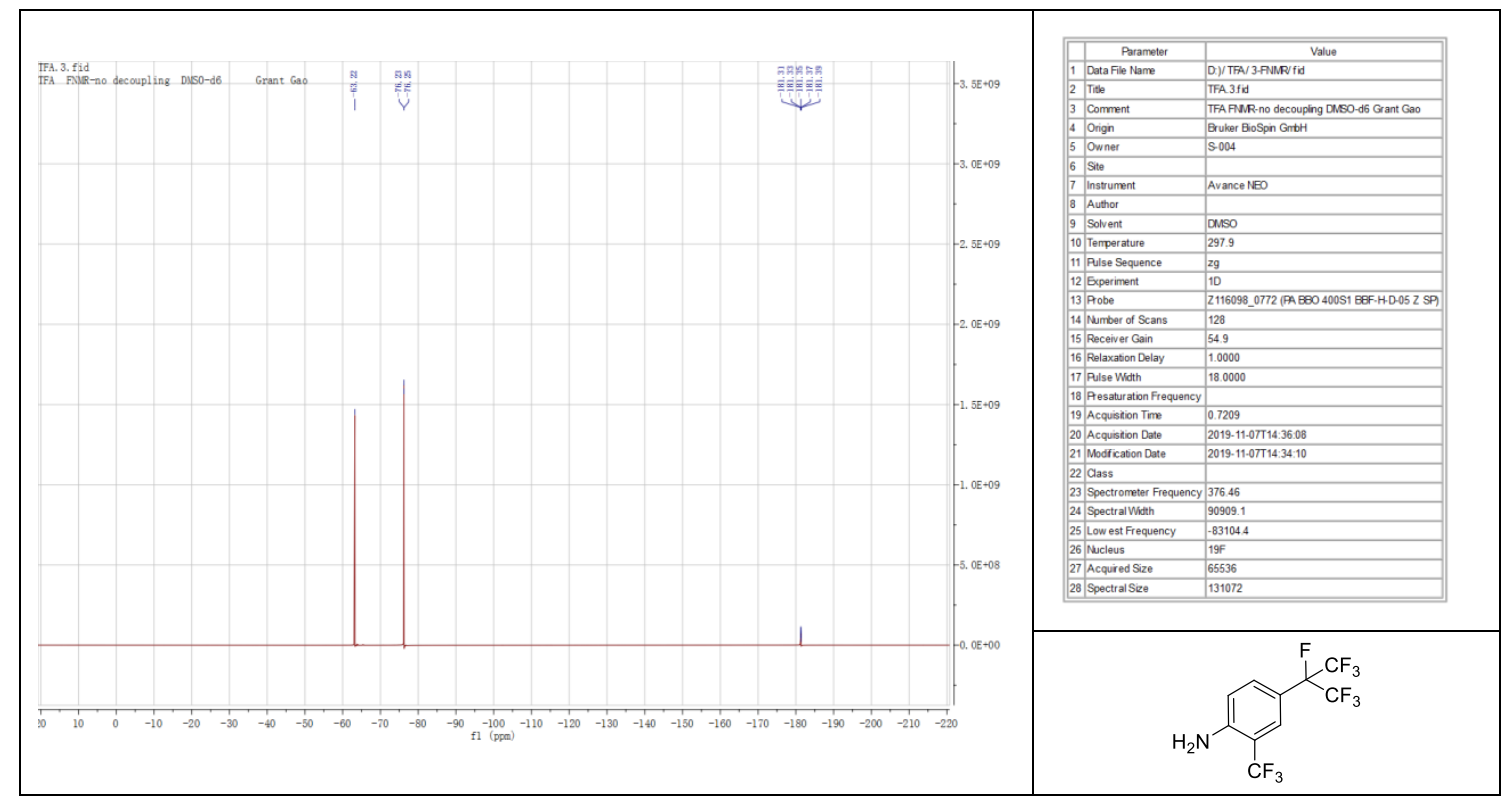


19. X-ray analysis of broflanilide (1).

Empirical formula

Formula weight

Temperature

Wavelength

Crystal system

Space group

Unit cell dimensions

Volume

Z

Density (calculated)

Absorption coefficient

$\mathrm{F}(000)$

Crystal size

Theta range for data collection

Index ranges

Reflections collected

Independent reflections

Completeness to theta $=66.591^{\circ}$

Absorption correction

Max. and min. transmission

Refinement method

Data / restraints / parameters

Goodness-of-fit on $\mathrm{F}^{2}$

Final R indices [I $>2 \operatorname{sigma}(\mathrm{I})]$

$\mathrm{R}$ indices (all data)

Extinction coefficient

Largest diff. peak and hole
$\mathrm{C}_{25} \mathrm{H}_{14} \mathrm{BrF}_{11} \mathrm{~N}_{2} \mathrm{O}_{2}$

663.29

293(2) K

$1.54184 \AA$

Monoclinic

P $121 / n 1$

$\mathrm{a}=15.1468(11) \AA$

$\mathrm{a}=90^{\circ}$.

$\mathrm{b}=9.5122(9) \AA$

$\mathrm{b}=105.660(8)^{\circ}$.

$\mathrm{c}=18.8339(16) \AA$

$\mathrm{g}=90^{\circ}$.

2612.8(4) $\AA^{3}$

4

$1.686 \mathrm{Mg} / \mathrm{m}^{3}$

$3.137 \mathrm{~mm}^{-1}$

1312

$0.3 \times 0.3 \times 0.3 \mathrm{~mm}^{3}$

5.251 to $66.591^{\circ}$.

$-18<=\mathrm{h}<=18,-10<=\mathrm{k}<=11,-22<=\mathrm{l}<=22$

33090

$4620[\mathrm{R}(\mathrm{int})=0.1195]$

$99.9 \%$

Semi-empirical from equivalents

1.00000 and 0.38285

Full-matrix least-squares on $\mathrm{F}^{2}$

4620 / 310 / 464

1.137

$\mathrm{R} 1=0.0739, \mathrm{wR} 2=0.2221$

$\mathrm{R} 1=0.0858, \mathrm{wR} 2=0.2494$

$\mathrm{n} / \mathrm{a}$

0.389 and -0.863 e. $\AA^{-3}$ 
Fractional atomic coordinates and equivalent isotropic displacement parameters $\left(\AA^{2} \times 10^{3}\right)$. U(eq) is defined as one third of the trace of the orthogonalized $\mathrm{U}^{\mathrm{ij}}$ tensor.

\begin{tabular}{|c|c|c|c|c|}
\hline & $\mathrm{x}$ & $\mathrm{y}$ & z & $\mathrm{U}(\mathrm{eq})$ \\
\hline $\operatorname{Br}(1)$ & $2648(1)$ & $4087(1)$ & 6994(1) & $108(1)$ \\
\hline $\mathrm{F}(1)$ & $437(1)$ & $3380(3)$ & $5347(2)$ & $81(1)$ \\
\hline $\mathrm{F}(4)$ & $2740(2)$ & $1684(4)$ & $4296(2)$ & $108(1)$ \\
\hline $\mathrm{N}(1)$ & $2174(2)$ & $2270(4)$ & $5601(2)$ & $70(1)$ \\
\hline $\mathrm{F}(3)$ & $4125(3)$ & $1055(4)$ & $4468(2)$ & $126(1)$ \\
\hline$C(21)$ & $3750(2)$ & $2153(4)$ & $5459(2)$ & $71(1)$ \\
\hline$C(11)$ & $826(2)$ & $1123(4)$ & $5849(2)$ & $63(1)$ \\
\hline $\mathrm{N}(2)$ & $-1355(2)$ & $3138(4)$ & $5336(2)$ & $74(1)$ \\
\hline $\mathrm{O}(2)$ & $-1671(2)$ & $5438(4)$ & $5383(2)$ & $97(1)$ \\
\hline $\mathrm{F}(2)$ & $3239(2)$ & $-60(3)$ & $4973(2)$ & $110(1)$ \\
\hline$C(20)$ & 4666(3) & $2517(5)$ & $5716(3)$ & $84(1)$ \\
\hline$C(18)$ & $4358(3)$ & $3819(5)$ & $6695(3)$ & $89(1)$ \\
\hline $\mathrm{F}(9)$ & $7365(8)$ & $3270(20)$ & $7439(9)$ & $123(4)$ \\
\hline$C(9)$ & $-732(2)$ & 2014(4) & $5579(2)$ & $66(1)$ \\
\hline$C(14)$ & $-1015(3)$ & $745(5)$ & $5806(2)$ & $75(1)$ \\
\hline$C(7)$ & $-1242(2)$ & $4409(5)$ & $5675(3)$ & $75(1)$ \\
\hline$C(15)$ & $1842(2)$ & $1237(5)$ & $5942(2)$ & $71(1)$ \\
\hline$C(13)$ & $-397(3)$ & $-329(5)$ & $6044(2)$ & $74(1)$ \\
\hline $\mathrm{C}(22)$ & $3454(3)$ & $1220(5)$ & $4788(3)$ & $83(1)$ \\
\hline$C(23)$ & $5939(5)$ & $3918(10)$ & $6699(5)$ & $86(2)$ \\
\hline$C(17)$ & $3448(3)$ & $3456(5)$ & $6449(2)$ & $76(1)$ \\
\hline$C(12)$ & $516(3)$ & $-135(4)$ & $6077(2)$ & $70(1)$ \\
\hline $\mathrm{O}(1)$ & $2353(2)$ & $407(4)$ & $6341(2)$ & $96(1)$ \\
\hline$C(5)$ & $-665(3)$ & $3590(5)$ & $6985(3)$ & $83(1)$ \\
\hline$C(19)$ & 4977(3) & $3353(5)$ & $6330(3)$ & $88(1)$ \\
\hline $\mathrm{F}(11)$ & $6205(11)$ & $2128(18)$ & $7553(11)$ & $150(6)$ \\
\hline $\mathrm{C}(1)$ & $605(4)$ & $4727(8)$ & 7811(4) & $116(2)$ \\
\hline$C(2)$ & $688(4)$ & $5601(7)$ & $7265(5)$ & $118(2)$ \\
\hline$C(10)$ & $183(2)$ & $2149(4)$ & $5591(2)$ & $65(1)$ \\
\hline$C(3)$ & $78(3)$ & $5515(5)$ & $6583(4)$ & $97(1)$ \\
\hline$C(16)$ & $3116(2)$ & 2619(4) & $5831(2)$ & $68(1)$ \\
\hline$C(4)$ & $-598(3)$ & $4490(4)$ & $6423(3)$ & $76(1)$ \\
\hline $\mathrm{C}(8)$ & $-2023(3)$ & $3009(7)$ & $4616(3)$ & $98(1)$ \\
\hline
\end{tabular}




\begin{tabular}{lllll}
$\mathrm{C}(6)$ & $-67(4)$ & $3714(7)$ & $7685(3)$ & $104(2)$ \\
$\mathrm{F}(10)$ & $6631(8)$ & $1890(20)$ & $6558(11)$ & $170(7)$ \\
$\mathrm{C}(24)$ & $6540(11)$ & $2834(18)$ & $7091(10)$ & $115(4)$ \\
$\mathrm{F}(7)$ & $7085(7)$ & $5359(15)$ & $6471(8)$ & $144(4)$ \\
$\mathrm{F}(8)$ & $5710(10)$ & $5845(13)$ & $5980(9)$ & $199(6)$ \\
$\mathrm{F}(6)$ & $6336(8)$ & $3985(15)$ & $5601(5)$ & $191(5)$ \\
$\mathrm{C}(25)$ & $6296(7)$ & $4750(11)$ & $6164(5)$ & $108(3)$ \\
$\mathrm{F}(5)$ & $6007(5)$ & $4890(10)$ & $7304(4)$ & $117(3)$ \\
$\mathrm{F}(8 \mathrm{~A})$ & $6385(9)$ & $1288(10)$ & $6880(9)$ & $150(5)$ \\
$\mathrm{F}(7 \mathrm{~A})$ & $6217(10)$ & $2750(20)$ & $7698(6)$ & $143(5)$ \\
$\mathrm{F}(6 \mathrm{~A})$ & $7427(8)$ & $2800(20)$ & $7323(11)$ & $126(5)$ \\
$\mathrm{F}(10 \mathrm{~A})$ & $5851(11)$ & $5480(20)$ & $5829(10)$ & $158(7)$ \\
$\mathrm{F}(9 \mathrm{~A})$ & $7175(8)$ & $5120(20)$ & $6536(13)$ & $118(6)$ \\
$\mathrm{F}(5 \mathrm{~A})$ & $6463(5)$ & $2965(15)$ & $5917(7)$ & $117(4)$ \\
$\mathrm{C}(23 \mathrm{~A})$ & $6077(8)$ & $3458(10)$ & $6482(6)$ & $80(3)$ \\
$\mathrm{C}(24 \mathrm{~A})$ & $6529(10)$ & $2565(16)$ & $7129(8)$ & $101(3)$ \\
$\mathrm{C}(25 \mathrm{~A})$ & $6290(9)$ & $4948(15)$ & $6501(8)$ & $101(4)$ \\
$\mathrm{F}(11 \mathrm{~A})$ & $6068(12)$ & $5512(17)$ & $7055(11)$ & $159(7)$ \\
\hline
\end{tabular}

Geometric parameters $\left(\AA^{2}\right)$ :

\begin{tabular}{ll}
\hline $\mathrm{Br}(1)-\mathrm{C}(17)$ & $1.884(4)$ \\
$\mathrm{F}(1)-\mathrm{C}(10)$ & $1.351(4)$ \\
$\mathrm{F}(4)-\mathrm{C}(22)$ & $1.298(6)$ \\
$\mathrm{N}(1)-\mathrm{C}(15)$ & $1.343(5)$ \\
$\mathrm{N}(1)-\mathrm{C}(16)$ & $1.414(5)$ \\
$\mathrm{F}(3)-\mathrm{C}(22)$ & $1.324(5)$ \\
$\mathrm{C}(21)-\mathrm{C}(20)$ & $1.383(6)$ \\
$\mathrm{C}(21)-\mathrm{C}(22)$ & $1.509(7)$ \\
$\mathrm{C}(21)-\mathrm{C}(16)$ & $1.405(5)$ \\
$\mathrm{C}(11)-\mathrm{C}(15)$ & $1.505(5)$ \\
$\mathrm{C}(11)-\mathrm{C}(12)$ & $1.394(5)$ \\
$\mathrm{C}(11)-\mathrm{C}(10)$ & $1.373(5)$ \\
$\mathrm{N}(2)-\mathrm{C}(9)$ & $1.418(5)$ \\
$\mathrm{N}(2)-\mathrm{C}(7)$ & $1.357(6)$ \\
$\mathrm{N}(2)-\mathrm{C}(8)$ & $1.461(6)$ \\
$\mathrm{O}(2)-\mathrm{C}(7)$ & $1.220(5)$
\end{tabular}




\begin{tabular}{|c|c|}
\hline $\mathrm{F}(2)-\mathrm{C}(22)$ & $1.332(6)$ \\
\hline $\mathrm{C}(20)-\mathrm{C}(19)$ & $1.378(7)$ \\
\hline $\mathrm{C}(18)-\mathrm{C}(17)$ & $1.374(6)$ \\
\hline C(18)-C(19) & $1.377(8)$ \\
\hline $\mathrm{F}(9)-\mathrm{C}(24)$ & $1.311(15)$ \\
\hline $\mathrm{C}(9)-\mathrm{C}(14)$ & $1.386(6)$ \\
\hline $\mathrm{C}(9)-\mathrm{C}(10)$ & $1.385(5)$ \\
\hline $\mathrm{C}(14)-\mathrm{C}(13)$ & $1.376(6)$ \\
\hline$C(7)-C(4)$ & $1.484(7)$ \\
\hline $\mathrm{C}(15)-\mathrm{O}(1)$ & $1.214(5)$ \\
\hline$C(13)-C(12)$ & $1.381(5)$ \\
\hline$C(23)-C(19)$ & $1.533(9)$ \\
\hline$C(23)-C(24)$ & $1.440(15)$ \\
\hline$C(23)-C(25)$ & $1.492(10)$ \\
\hline$C(23)-F(5)$ & $1.450(13)$ \\
\hline$C(17)-C(16)$ & $1.387(6)$ \\
\hline $\mathrm{C}(5)-\mathrm{C}(4)$ & $1.385(7)$ \\
\hline$C(5)-C(6)$ & $1.390(8)$ \\
\hline$C(19)-C(23 A)$ & $1.617(13)$ \\
\hline $\mathrm{F}(11)-\mathrm{C}(24)$ & $1.306(15)$ \\
\hline$C(1)-C(2)$ & $1.354(10)$ \\
\hline$C(1)-C(6)$ & $1.375(9)$ \\
\hline $\mathrm{C}(2)-\mathrm{C}(3)$ & $1.368(10)$ \\
\hline$C(3)-C(4)$ & $1.388(6)$ \\
\hline $\mathrm{F}(10)-\mathrm{C}(24)$ & $1.378(16)$ \\
\hline $\mathrm{F}(7)-\mathrm{C}(25)$ & $1.314(10)$ \\
\hline $\mathrm{F}(8)-\mathrm{C}(25)$ & $1.351(11)$ \\
\hline $\mathrm{F}(6)-\mathrm{C}(25)$ & $1.301(10)$ \\
\hline $\mathrm{F}(8 \mathrm{~A})-\mathrm{C}(24 \mathrm{~A})$ & $1.299(15)$ \\
\hline $\mathrm{F}(7 \mathrm{~A})-\mathrm{C}(24 \mathrm{~A})$ & $1.293(14)$ \\
\hline $\mathrm{F}(6 \mathrm{~A})-\mathrm{C}(24 \mathrm{~A})$ & $1.329(14)$ \\
\hline$F(10 A)-C(25 A)$ & $1.357(12)$ \\
\hline $\mathrm{F}(9 \mathrm{~A})-\mathrm{C}(25 \mathrm{~A})$ & $1.335(12)$ \\
\hline $\mathrm{F}(5 \mathrm{~A})-\mathrm{C}(23 \mathrm{~A})$ & $1.423(16)$ \\
\hline$C(23 \mathrm{~A})-\mathrm{C}(24 \mathrm{~A})$ & $1.492(14)$ \\
\hline$C(23 \mathrm{~A})-\mathrm{C}(25 \mathrm{~A})$ & $1.451(14)$ \\
\hline $\mathrm{C}(25 \mathrm{~A})-\mathrm{F}(11 \mathrm{~A})$ & $1.296(12)$ \\
\hline
\end{tabular}




\begin{tabular}{|c|c|}
\hline$C(15)-N(1)-C(16)$ & $120.1(3)$ \\
\hline $\mathrm{C}(20)-\mathrm{C}(21)-\mathrm{C}(22)$ & $118.9(4)$ \\
\hline$C(20)-C(21)-C(16)$ & $120.2(4)$ \\
\hline $\mathrm{C}(16)-\mathrm{C}(21)-\mathrm{C}(22)$ & $120.9(3)$ \\
\hline $\mathrm{C}(12)-\mathrm{C}(11)-\mathrm{C}(15)$ & $116.4(3)$ \\
\hline $\mathrm{C}(10)-\mathrm{C}(11)-\mathrm{C}(15)$ & 126.6(3) \\
\hline $\mathrm{C}(10)-\mathrm{C}(11)-\mathrm{C}(12)$ & $117.0(3)$ \\
\hline $\mathrm{C}(9)-\mathrm{N}(2)-\mathrm{C}(8)$ & $118.0(4)$ \\
\hline $\mathrm{C}(7)-\mathrm{N}(2)-\mathrm{C}(9)$ & 122.1(3) \\
\hline $\mathrm{C}(7)-\mathrm{N}(2)-\mathrm{C}(8)$ & $118.7(4)$ \\
\hline$C(19)-C(20)-C(21)$ & $121.4(4)$ \\
\hline $\mathrm{C}(17)-\mathrm{C}(18)-\mathrm{C}(19)$ & $120.4(5)$ \\
\hline $\mathrm{C}(14)-\mathrm{C}(9)-\mathrm{N}(2)$ & $121.5(3)$ \\
\hline $\mathrm{C}(10)-\mathrm{C}(9)-\mathrm{N}(2)$ & $120.7(4)$ \\
\hline$C(10)-C(9)-C(14)$ & $117.8(4)$ \\
\hline $\mathrm{C}(13)-\mathrm{C}(14)-\mathrm{C}(9)$ & $120.5(3)$ \\
\hline $\mathrm{N}(2)-\mathrm{C}(7)-\mathrm{C}(4)$ & $117.4(4)$ \\
\hline $\mathrm{O}(2)-\mathrm{C}(7)-\mathrm{N}(2)$ & $121.2(4)$ \\
\hline $\mathrm{O}(2)-\mathrm{C}(7)-\mathrm{C}(4)$ & $121.3(4)$ \\
\hline $\mathrm{N}(1)-\mathrm{C}(15)-\mathrm{C}(11)$ & $120.0(3)$ \\
\hline $\mathrm{O}(1)-\mathrm{C}(15)-\mathrm{N}(1)$ & $120.9(3)$ \\
\hline $\mathrm{O}(1)-\mathrm{C}(15)-\mathrm{C}(11)$ & 119.1(4) \\
\hline$C(14)-C(13)-C(12)$ & $120.0(4)$ \\
\hline $\mathrm{F}(4)-\mathrm{C}(22)-\mathrm{F}(3)$ & $108.0(4)$ \\
\hline $\mathrm{F}(4)-\mathrm{C}(22)-\mathrm{C}(21)$ & $113.6(4)$ \\
\hline $\mathrm{F}(4)-\mathrm{C}(22)-\mathrm{F}(2)$ & $106.4(4)$ \\
\hline $\mathrm{F}(3)-\mathrm{C}(22)-\mathrm{C}(21)$ & 111.1(4) \\
\hline $\mathrm{F}(3)-\mathrm{C}(22)-\mathrm{F}(2)$ & $106.4(4)$ \\
\hline $\mathrm{F}(2)-\mathrm{C}(22)-\mathrm{C}(21)$ & $111.0(4)$ \\
\hline $\mathrm{C}(24)-\mathrm{C}(23)-\mathrm{C}(19)$ & $112.0(9)$ \\
\hline $\mathrm{C}(24)-\mathrm{C}(23)-\mathrm{C}(25)$ & $115.6(10)$ \\
\hline $\mathrm{C}(24)-\mathrm{C}(23)-\mathrm{F}(5)$ & $98.8(9)$ \\
\hline$C(25)-C(23)-C(19)$ & $110.7(7)$ \\
\hline $\mathrm{F}(5)-\mathrm{C}(23)-\mathrm{C}(19)$ & $115.9(7)$ \\
\hline $\mathrm{F}(5)-\mathrm{C}(23)-\mathrm{C}(25)$ & 103.3(8) \\
\hline $\mathrm{C}(18)-\mathrm{C}(17)-\mathrm{Br}(1)$ & $118.0(4)$ \\
\hline $\mathrm{C}(18)-\mathrm{C}(17)-\mathrm{C}(16)$ & $122.0(4)$ \\
\hline $\mathrm{C}(16)-\mathrm{C}(17)-\mathrm{Br}(1)$ & $119.9(3)$ \\
\hline
\end{tabular}




\begin{tabular}{|c|c|}
\hline$C(13)-C(12)-C(11)$ & $121.2(4)$ \\
\hline$C(4)-C(5)-C(6)$ & $120.6(5)$ \\
\hline$C(20)-C(19)-C(23)$ & $130.6(6)$ \\
\hline$C(20)-C(19)-C(23 A)$ & $106.7(6)$ \\
\hline$C(18)-C(19)-C(20)$ & $118.7(4)$ \\
\hline $\mathrm{C}(18)-\mathrm{C}(19)-\mathrm{C}(23)$ & $110.7(6)$ \\
\hline $\mathrm{C}(18)-\mathrm{C}(19)-\mathrm{C}(23 \mathrm{~A})$ & $134.5(6)$ \\
\hline$C(2)-C(1)-C(6)$ & $121.1(6)$ \\
\hline$C(1)-C(2)-C(3)$ & $119.9(5)$ \\
\hline $\mathrm{F}(1)-\mathrm{C}(10)-\mathrm{C}(11)$ & $119.8(3)$ \\
\hline $\mathrm{F}(1)-\mathrm{C}(10)-\mathrm{C}(9)$ & $116.8(3)$ \\
\hline$C(11)-C(10)-C(9)$ & $123.4(4)$ \\
\hline$C(2)-C(3)-C(4)$ & $121.1(6)$ \\
\hline $\mathrm{C}(21)-\mathrm{C}(16)-\mathrm{N}(1)$ & $122.8(4)$ \\
\hline $\mathrm{C}(17)-\mathrm{C}(16)-\mathrm{N}(1)$ & $119.9(4)$ \\
\hline $\mathrm{C}(17)-\mathrm{C}(16)-\mathrm{C}(21)$ & $117.2(4)$ \\
\hline$C(5)-C(4)-C(7)$ & $121.9(4)$ \\
\hline$C(5)-C(4)-C(3)$ & $118.1(5)$ \\
\hline$C(3)-C(4)-C(7)$ & $120.0(5)$ \\
\hline$C(1)-C(6)-C(5)$ & $119.0(6)$ \\
\hline $\mathrm{F}(9)-\mathrm{C}(24)-\mathrm{C}(23)$ & $114.7(13)$ \\
\hline $\mathrm{F}(9)-\mathrm{C}(24)-\mathrm{F}(10)$ & $107.4(13)$ \\
\hline $\mathrm{F}(11)-\mathrm{C}(24)-\mathrm{F}(9)$ & $108.5(14)$ \\
\hline $\mathrm{F}(11)-\mathrm{C}(24)-\mathrm{C}(23)$ & $113.9(13)$ \\
\hline $\mathrm{F}(11)-\mathrm{C}(24)-\mathrm{F}(10)$ & $106.5(14)$ \\
\hline $\mathrm{F}(10)-\mathrm{C}(24)-\mathrm{C}(23)$ & $105.3(12)$ \\
\hline $\mathrm{F}(7)-\mathrm{C}(25)-\mathrm{C}(23)$ & $112.8(10)$ \\
\hline $\mathrm{F}(7)-\mathrm{C}(25)-\mathrm{F}(8)$ & $103.3(10)$ \\
\hline $\mathrm{F}(8)-\mathrm{C}(25)-\mathrm{C}(23)$ & $104.5(9)$ \\
\hline $\mathrm{F}(6)-\mathrm{C}(25)-\mathrm{C}(23)$ & $111.0(9)$ \\
\hline $\mathrm{F}(6)-\mathrm{C}(25)-\mathrm{F}(7)$ & $111.6(9)$ \\
\hline $\mathrm{F}(6)-\mathrm{C}(25)-\mathrm{F}(8)$ & $113.3(11)$ \\
\hline $\mathrm{F}(5 \mathrm{~A})-\mathrm{C}(23 \mathrm{~A})-\mathrm{C}(19)$ & 117.3(9) \\
\hline$F(5 A)-C(23 A)-C(24 A)$ & $103.5(10)$ \\
\hline$F(5 A)-C(23 A)-C(25 A)$ & $102.0(10)$ \\
\hline$C(24 A)-C(23 A)-C(19)$ & 109.4(9) \\
\hline$C(25 A)-C(23 A)-C(19)$ & $106.0(8)$ \\
\hline$C(25 A)-C(23 A)-C(24 A)$ & $119.0(11)$ \\
\hline
\end{tabular}




$\begin{array}{ll}F(8 A)-C(24 A)-F(6 A) & 108.3(12) \\ F(8 A)-C(24 A)-C(23 A) & 104.0(11) \\ F(7 A)-C(24 A)-F(8 A) & 111.2(13) \\ F(7 A)-C(24 A)-F(6 A) & 109.0(12) \\ F(7 A)-C(24 A)-C(23 A) & 114.3(13) \\ F(6 A)-C(24 A)-C(23 A) & 109.9(12) \\ F(10 A)-C(25 A)-C(23 A) & 106.7(12) \\ F(9 A)-C(25 A)-F(10 A) & 103.5(11) \\ F(9 A)-C(25 A)-C(23 A) & 109.5(13) \\ F(11 A)-C(25 A)-F(10 A) & 115.5(15) \\ F(11 A)-C(25 A)-F(9 A) & 112.6(14) \\ F(11 A)-C(25 A)-C(23 A) & 108.7(12)\end{array}$

Symmetry transformations used to generate equivalent atoms:

$\left(\AA^{2} \mathrm{x} 10^{3}\right)$. The anisotropic displacement factor exponent takes the form: $-2 \mathrm{p}^{2}\left[\mathrm{~h}^{2} \mathrm{a}^{* 2} \mathrm{U}^{11}+\ldots+\right.$ $2 \mathrm{~h} \mathrm{k} \mathrm{a}^{*} \mathrm{~b}^{*} \mathrm{U}^{12}$ ]

\begin{tabular}{lcccccc}
\hline & $\mathrm{U}^{11}$ & $\mathrm{U}^{22}$ & $\mathrm{U}^{33}$ & $\mathrm{U}^{23}$ & $\mathrm{U}^{13}$ & $\mathrm{U}^{12}$ \\
\hline $\mathrm{Br}(1)$ & $107(1)$ & $120(1)$ & $102(1)$ & $-9(1)$ & $38(1)$ & $20(1)$ \\
$\mathrm{F}(1)$ & $60(1)$ & $79(2)$ & $113(2)$ & $26(1)$ & $38(1)$ & $10(1)$ \\
$\mathrm{F}(4)$ & $107(2)$ & $120(2)$ & $93(2)$ & $-4(2)$ & $22(2)$ & $3(2)$ \\
$\mathrm{N}(1)$ & $50(1)$ & $81(2)$ & $81(2)$ & $15(2)$ & $19(1)$ & $7(1)$ \\
$\mathrm{F}(3)$ & $103(2)$ & $144(3)$ & $154(3)$ & $-41(2)$ & $75(2)$ & $-16(2)$ \\
$\mathrm{C}(21)$ & $55(2)$ & $66(2)$ & $95(2)$ & $9(2)$ & $26(2)$ & $2(2)$ \\
$\mathrm{C}(11)$ & $52(2)$ & $71(2)$ & $69(2)$ & $0(2)$ & $22(1)$ & $-1(1)$ \\
$\mathrm{N}(2)$ & $52(1)$ & $84(2)$ & $84(2)$ & $4(2)$ & $18(1)$ & $7(1)$ \\
$\mathrm{O}(2)$ & $82(2)$ & $91(2)$ & $131(3)$ & $38(2)$ & $49(2)$ & $23(2)$ \\
$\mathrm{F}(2)$ & $113(2)$ & $80(2)$ & $141(3)$ & $-7(2)$ & $38(2)$ & $-16(2)$ \\
$\mathrm{C}(20)$ & $56(2)$ & $84(3)$ & $117(3)$ & $21(2)$ & $34(2)$ & $1(2)$ \\
$\mathrm{C}(18)$ & $76(2)$ & $87(3)$ & $95(3)$ & $13(2)$ & $11(2)$ & $-14(2)$ \\
$\mathrm{F}(9)$ & $59(4)$ & $175(12)$ & $129(7)$ & $7(7)$ & $16(4)$ & $-1(5)$ \\
$\mathrm{C}(9)$ & $51(2)$ & $77(2)$ & $71(2)$ & $-4(2)$ & $18(1)$ & $1(2)$ \\
$\mathrm{C}(14)$ & $57(2)$ & $84(3)$ & $88(3)$ & $-7(2)$ & $26(2)$ & $-7(2)$ \\
$\mathrm{C}(7)$ & $53(2)$ & $77(2)$ & $103(3)$ & $10(2)$ & $38(2)$ & $5(2)$ \\
$\mathrm{C}(15)$ & $52(2)$ & $85(3)$ & $77(2)$ & $7(2)$ & $21(2)$ & $6(2)$ \\
$\mathrm{C}(13)$ & $68(2)$ & $72(2)$ & $86(3)$ & $0(2)$ & $27(2)$ & $-7(2)$ \\
& & & & & & \\
& & & & 26 & & \\
\end{tabular}




$\begin{array}{lcccccc}\mathrm{C}(22) & 68(2) & 86(3) & 107(3) & 4(2) & 41(2) & 1(2) \\ \mathrm{C}(23) & 56(3) & 98(5) & 105(5) & 2(4) & 23(3) & -8(3) \\ \mathrm{C}(17) & 65(2) & 77(3) & 86(2) & 10(2) & 19(2) & 7(2) \\ \mathrm{C}(12) & 64(2) & 69(2) & 80(2) & 4(2) & 24(2) & 2(2) \\ \mathrm{O}(1) & 57(1) & 105(2) & 123(3) & 47(2) & 21(2) & 13(2) \\ \mathrm{C}(5) & 70(2) & 90(3) & 93(3) & -13(2) & 26(2) & -11(2) \\ \mathrm{C}(19) & 61(2) & 84(3) & 112(3) & 25(2) & 12(2) & -14(2) \\ \mathrm{F}(11) & 100(6) & 144(10) & 195(11) & 76(8) & 18(7) & -20(6) \\ \mathrm{C}(1) & 73(3) & 149(5) & 127(4) & -64(4) & 26(3) & -12(3) \\ \mathrm{C}(2) & 78(3) & 116(4) & 176(5) & -74(4) & 62(3) & -35(3) \\ \mathrm{C}(10) & 57(2) & 68(2) & 73(2) & 2(2) & 23(2) & -1(2) \\ \mathrm{C}(3) & 79(2) & 80(3) & 145(4) & -26(3) & 55(3) & -16(2) \\ \mathrm{C}(16) & 51(2) & 66(2) & 86(2) & 17(2) & 19(2) & 5(1) \\ \mathrm{C}(4) & 57(2) & 70(2) & 107(3) & -11(2) & 35(2) & 1(2) \\ \mathrm{C}(8) & 73(2) & 128(4) & 89(3) & 9(3) & 12(2) & 15(3) \\ \mathrm{C}(6) & 100(3) & 116(4) & 97(3) & -17(3) & 27(3) & -3(3) \\ \mathrm{F}(10) & 103(6) & 147(11) & 242(12) & -49(10) & 19(7) & 21(6) \\ \mathrm{C}(24) & 66(6) & 136(7) & 137(8) & 22(5) & 17(5) & 3(5) \\ \mathrm{F}(7) & 120(6) & 138(7) & 183(9) & -14(6) & 55(6) & -71(6) \\ \mathrm{F}(8) & 167(9) & 129(7) & 285(13) & 73(6) & 35(8) & -17(5) \\ \mathrm{F}(6) & 176(7) & 275(11) & 154(7) & -65(7) & 99(6) & -108(8) \\ \mathrm{C}(25) & 91(5) & 130(6) & 104(5) & 7(4) & 32(4) & -31(4) \\ \mathrm{F}(5) & 94(4) & 150(7) & 120(5) & -38(4) & 50(3) & -26(4) \\ \mathrm{F}(8 \mathrm{~A}) & 117(7) & 101(5) & 213(11) & 17(6) & 13(6) & 11(5) \\ \mathrm{F}(7 \mathrm{~A}) & 91(5) & 239(14) & 102(5) & 15(6) & 33(4) & 5(8) \\ \mathrm{F}(6 \mathrm{~A}) & 52(3) & 155(10) & 167(10) & 28(7) & 24(4) & 3(4) \\ \mathrm{F}(10 \mathrm{~A}) & 92(7) & 151(12) & 210(11) & 108(10) & 5(7) & -42(8) \\ \mathrm{F}(9 \mathrm{~A}) & 60(5) & 123(10) & 171(13) & 14(9) & 33(6) & -20(5) \\ \mathrm{F}(5 \mathrm{~A}) & 75(4) & 150(8) & 134(6) & -46(6) & 43(4) & -8(5) \\ \mathrm{C}(23 \mathrm{~A}) & 71(4) & 76(5) & 96(6) & -1(4) & 28(4) & -6(4) \\ \mathrm{C}(24 \mathrm{~A}) & 61(5) & 115(6) & 123(6) & 19(6) & 18(5) & -4(5) \\ \mathrm{C}(25 \mathrm{~A}) & 85(6) & 99(6) & 120(8) & -1(6) & 31(6) & -29(6) \\ \mathrm{F}(11 \mathrm{~A}) & 128(9) & 135(11) & 236(13) & -65(10) & 88(9) & -23(8)\end{array}$


Displacement parameters $\left(\AA^{2} \times 10^{3}\right)$.

\begin{tabular}{lrrrc}
\hline & $\mathrm{x}$ & $\mathrm{y}$ & $\mathrm{z}$ & $\mathrm{U}(\mathrm{eq})$ \\
\hline $\mathrm{H}(1)$ & 1813 & 2720 & 5242 & 84 \\
$\mathrm{H}(20)$ & 5080 & 2191 & 5468 & 100 \\
$\mathrm{H}(18)$ & 4557 & 4383 & 7111 & 106 \\
$\mathrm{H}(14)$ & -1626 & 619 & 5798 & 90 \\
$\mathrm{H}(13)$ & -595 & -1187 & 6182 & 89 \\
$\mathrm{H}(12)$ & 933 & -855 & 6254 & 84 \\
$\mathrm{H}(5)$ & -1115 & 2898 & 6892 & 100 \\
$\mathrm{H}(1 \mathrm{~A})$ & 1008 & 4813 & 8278 & 140 \\
$\mathrm{H}(2)$ & 1160 & 6259 & 7354 & 142 \\
$\mathrm{H}(3)$ & 118 & 6156 & 6219 & 116 \\
$\mathrm{H}(8 \mathrm{~A})$ & -1790 & 3466 & 4249 & 148 \\
$\mathrm{H}(8 \mathrm{~B})$ & -2589 & 3443 & 4633 & 148 \\
$\mathrm{H}(8 \mathrm{C})$ & -2127 & 2032 & 4493 & 148 \\
$\mathrm{H}(6)$ & -120 & 3120 & 8064 & 125 \\
& & & & \\
\hline
\end{tabular}

